\title{
Electromagnetic Finite Elements \\ Based on a Four-Potential \\ Variational Principle
}

James Schuler and Carlos A. Felippa

University of Colorado

Boulder, Colorado

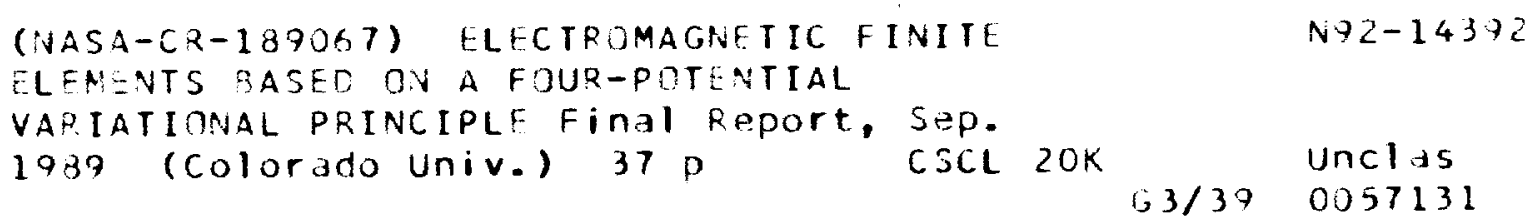

November 1991

Prepared for

Lewis Research Center

Under Grant NAG3-934

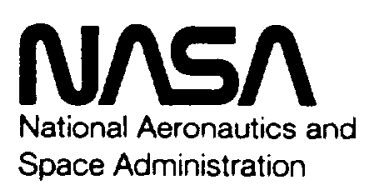





\title{
ELECTROMAGNETIC FINITE ELEMENTS BASED ON A FOUR-POTENTIAL VARIATIONAL PRINCIPLE
}

\author{
JAMES SCHULER \\ CARLOS A. FELIPPA \\ Department of Aerospace Engineering Sciences \\ and Center for Space Structures and Controls \\ University of Colorado \\ Boulder, Colorado 80909-0429, USA
}

\begin{abstract}
SUMMARY
We derive electromagnetic finite elements based on a variational principle that uses the electromagnetic four-potential as primary variable. This choice is used to construct elements suitable for downstream coupling with mechanical and thermal finite elements for the analysis of electromagnetic/mechanical systems that involve superconductors. The key advantages of the four-potential are: the number of degrees of freedom per node remain modest as the problem dimensionality increases, jump discontinuities on interfaces are naturally accomodated, and static as well as dynamics are included without any a priori approximations. The new elements are tested on an axisymmetric problem under steady-state forcing conditions. The results are in excellent agreement with analytical solutions.
\end{abstract}




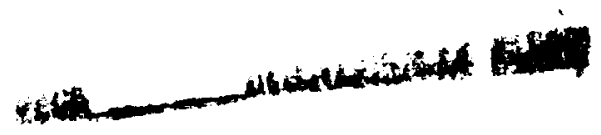




\section{INTRODUCTION}

The present work is part of a research program for the numerical simulation of electromagnetic/mechanical systems that involve superconductors. The simulation involves the interaction of the following four components:

(1) Mechanical Fields: displacements, stresses, strains and mechanical forces.

(2) Thermal Fields: temperature and heat fluxes.

(3) Electromagnetic (EM) Fields: electric and magnetic field strengths and fluxes, currents and charges.

(4) Coupling Fields: the foundamental coupling effect is the constitutive behavior of the materials involved. Particularly important are the metallurgical phase change phenomena triggered by thermal, mechanical and EM fields.

\subsection{Finite Element Treatment}

The first three fields (mechanical, thermal and electromagnetic) are treated by the finite element method. This treatment produces the spatial discretization of the continuum into mechanical, thermal and electromagnetic meshes of finite number of degrees of freedom. The finite element discretization may be developed in two ways:

(1) Simultaneous Treatment. The whole problem is treated as an indivisible whole. The three meshes noted above become tightly coupled, with common nodes and elements.

(2) Staged Treatment. The mechanical, thermal and electromagnetic components of the problem are treated separately. Finite element meshes for these components may be developed separately. Coupling effects are viewed as information that has to be transferred between these three meshes.

The present research follows the staged treatment. More specifically, we develop finite element models for the fields in isolation, and then treat coupling effects as interaction forces between these models. This "divide and conquer" strategy is ingrained in the partitioned treatment of coupled problems $[4,16]$, which offers significant advantages in terms of computational efficiency and software modularity. Another advantage relates to the way research into complex problems can be made more productive. It centers on the observation that some aspects of the problem are either better understood or less physically relevant than others. These aspects may be then temporarily left alone while efforts are concentrated on the less developed and/or more physically important aspects. The staged treatment is better suited to this approach.

\subsection{Mechanical Elements}

Mechanical elements for this research have been derived using general variational principles that decouple the element boundary from the interior thus providing efficient ways to work 
out coupling with non-mechanical fields. The point of departure was previous research into the free-formulation variational principles reported in Ref. [5]. A more general formulation for the mechanical elements, which includes the assumed natural strain formulation, was established and reported in Refs. $[5,6,14,15]$. New representations of thermal fields have not been addressed as standard formulations are considered adequate for the coupled-field phases of this research.

\section{ELECTROMAGNETIC ELEMENTS}

The development of electromagnetic (EM) finite elements has not received to date the same degree of attention given to mechanical and thermal elements. Part of the reason is the widespread use of analytical and semianalytical methods in electrical engineering. These methods have been highly refined for specialized but important problems such as circuits and waveguides. Thus the advantages of finite elements in terms of generality have not been enough to counterweight established techniques. Much of the EM finite element work to date has been done in England and is well described in the surveys by Davies [1] and Trowbridge [21]. The general impression conveyed by these surveys is one of an unsettled subject, reminiscent of the early period (1960-1970) of finite elements in structural mechanics. A great number of formulations that combine flux, intensity, and scalar potentials are described with the recommended choice varying according to the application, medium involved (polarizable, dielectric, semiconductors, etc.) number of space dimensions, time-dependent characteristics (static, quasi-static, harmonic or transient) as well as other factors of lesser importance. The possibility of a general variational formulation has not apparently been recognized.

In the present work, the derivation of electromagnetic (EM) elements is based on a variational formulation that uses the four-potential as primary variable. The electric field is represented by a scalar potential and the magnetic field by a vector potential. The formulation of these variational principle proceeds along lines previously developed for the acoustic fluid problem [7,8].

The main advantages of using potentials as primary variables as opposed to the more conventional EM finite elements based on intensity and/or flux fields are, in order or importance:

1. Interface discontinuities are automatically taken care of without any special intervention.

2. No approximations are invoked a priori since the general Maxwell equations are used.

3. The number of degrees of freedom per finite element node is kept modest as the problem dimensionality increases.

4. Coupling with the mechanical and thermal fields, which involves derived fields, can be naturally evaluated at the Gauss points at which derivatives of the potentials are evaluated.

Following a recapitulation of the basic field equations, the variational principle is stated. 
The discretization of these principle into finite element equations produces semidiscrete dynamical equations, which are specialized to the axisymmetric case. These equations are validated in a simulation of a cylindrical conductor wire.

\section{ELECTROMAGNETIC FIELD EQUATIONS}

\subsection{The Maxwell Equations}

The original Maxwell equations (1873) involve four spatial fields: $B, D, \mathbf{E}$ and $\mathbf{H}$. Vectors $\mathbf{E}$ and $\mathbf{H}$ represents the electric and magnetic field strengths (also called intensities), respectively, whereas $\mathbf{D}$ and $\mathbf{B}$ represent the electric and magnetic flux densities, respectively. All of these are three-vector quantities, that is, vector fields in three-dimensional space $\left(x_{1} \equiv x, x_{2} \equiv y, x_{3} \equiv z\right)$ :

$$
\mathbf{E}=\left\{\begin{array}{l}
E_{1} \\
E_{2} \\
E_{3}
\end{array}\right\}, \quad \mathbf{D}=\left\{\begin{array}{l}
D_{1} \\
D_{2} \\
D_{3}
\end{array}\right\}, \quad \mathbf{E}=\left\{\begin{array}{l}
E_{1} \\
E_{2} \\
E_{3}
\end{array}\right\}, \quad \mathbf{H}=\left\{\begin{array}{l}
H_{1} \\
H_{2} \\
H_{3}
\end{array}\right\} .
$$

Other quantities are the electric current 3-vector $j$ and the electric charge density $\rho$ (a scalar). Units for these and other quantities of interest in this work are summarized in Tables 1-2.

With this notation, and using superposed dots to denote differentiation with respect to time $t$, we can state Maxwell equations as*

$$
\begin{aligned}
\dot{\mathbf{B}}+\nabla \times \mathbf{E} & =\mathbf{0}, & & \nabla \times \mathbf{H}-\dot{\mathbf{D}}=\mathbf{j}, \\
\nabla \cdot \mathbf{D} & =\rho, & & \nabla \cdot \mathbf{B}=0 .
\end{aligned}
$$

The first and second equation are also known as Faraday's and Ampère-Maxwell laws, respectively.

The system (2) supplies a total of eight partial differential equations, which as stated are independent of the properties of the underlying medium.

\subsection{Constitutive Equations}

The field intensities $\mathbf{E}$ and $\mathbf{H}$ and the corresponding flux densities $\mathbf{D}$ and $\mathbf{B}$ are not independent but are connected by the electromagnetic constitutive equations. For an electromagnetically isotropic, non-polarized material the equations are

$$
\mathrm{B}=\mu \mathrm{H}, \quad \mathrm{D}=\epsilon \mathbf{E}
$$

* Some authors, for example Eyges [2], include $4 \pi$ factors and the speed of light $c$ in the Maxwell equations. Other textbooks, e.g. $[19,20]$, follow Heaviside's advice in using technical units that eliminate such confusing factors. 
Inble 1 Mectric and Magnetic Quantities

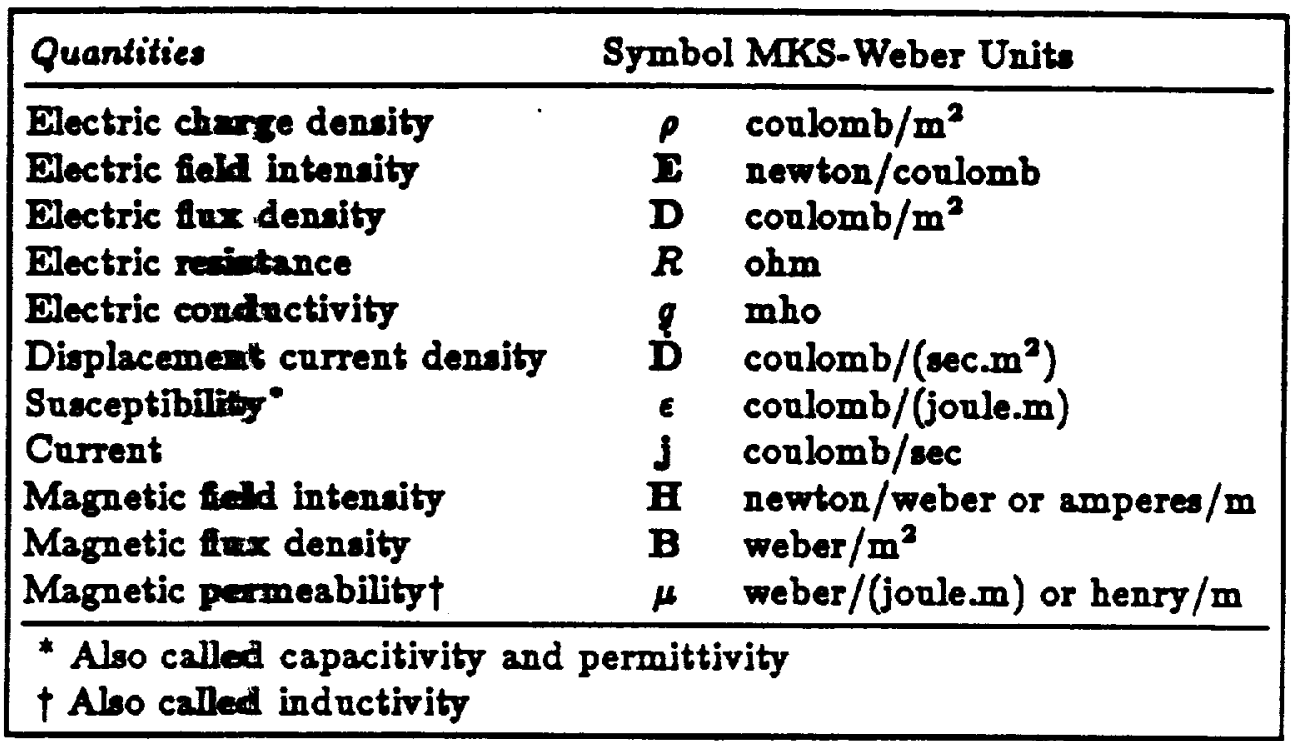

Table 2 Bquivalencea Between Various MKS-Giorgi Unita

1 newton $\equiv 1$ kg.m/sec
1 joule $\equiv 1$ newton.m
1 watt $\equiv 1$ joule/sec
1 coulomb $\equiv 1$ ampere.sec
1 ohm $\equiv 1$ volt $/$ ampere
1 farad $\equiv 1$ coulomb $/$ rolt
1 henry $\equiv 1$ (volt.sec)/ampere
1 weber $\equiv 1 \mathrm{rolt} . \sec$
1 mho $\equiv 1 \mathrm{ohm}^{-1}$

where $\mu$ and $\epsilon$ are the permeability and susceptibility, respectively, of the material $\uparrow$. These coefficients are functions of position but (for static or harmonic fields) do not depend on time. In the general case of a non-isotropic material both $\mu$ and $\epsilon$ become tensors. Even in isotropic media $\mu$ in general is a complicated function of $\mathbf{H}$; in ferromagnetic materials it depends on the previoas history (hysteresis effect).

In free space $\mu=\mu_{0}$ and $\epsilon=\epsilon_{0}$, which are connected by

$$
c_{0}^{2}=\frac{1}{\mu_{0} \epsilon_{0}}
$$

where $c_{0}$ is the speed of hight in a free vacuum. In MKS-A units, $c_{0}=3.10^{9} \mathrm{~m} / \mathrm{sec}$ and

$$
\mu_{0}=4 \pi \times 10^{-7} \text { henry } / \mathrm{m}, \epsilon_{0}=\mu_{0}^{-1} c_{0}^{-2}=(36 \pi)^{-1} \times 10^{-11} \mathrm{sec}^{2} /(\text { henry.m) }
$$

† Other names are ofter used, see Table 1. 
The condition $\mu \approx \mu_{0}$ holds well for most practical purposes in such media as air and copper; in fact $\mu_{\text {air }}=1.0000004 \mu_{0}$ and $\mu_{\text {copper }}=.99999 \mu_{0}$.

The electrical field strength $\mathbf{E}$ is further related to the current density $\mathbf{j}$ by Ohm's law:

$$
\mathrm{j}=\boldsymbol{g} \mathbf{E}
$$

where $g$ is the conductivity of the material. Again for an non-isotropic material $g$ is generally a tensor which may also contain real and imaginary components; in which case the above relation becomes the generalized Ohm's law. For good conductors $g>>\epsilon$; for bad conductors $g<<\epsilon$. In free space, $g=0$.

\section{S.S Maxwell Equations in Terms of $\mathbf{E}$ and $\mathbf{B}$}

To pass to the four-potential considered in Section 4 it is convenient to express Maxwell's equations in terms of the electrical field strength $\mathbf{E}$ and the magnetic flux $\mathbf{B}$. In fact this is the pair most frequently used in electromagnetic work that involve arbitrary media. On eliminating $D$ and $H$ through the constitutive equations (3) we obtain

$$
\begin{aligned}
\dot{\mathbf{B}}+\nabla \times \mathbf{E}=0, & \nabla \times \mathbf{B}-\mu \epsilon \dot{\mathbf{E}}=\mu \mathbf{j}, \\
\nabla \cdot \mathbf{E}=\rho / \epsilon, & \nabla \cdot \mathbf{B}=0 .
\end{aligned}
$$

The second equation assumes that $\epsilon$ is independent of time; otherwise $\epsilon \dot{\mathrm{E}}=\epsilon d \mathrm{E} / d t$ should be replaced by $d(\epsilon \mathbf{E}) / d t$. In charge-free vacuum the equations reduce to

$$
\begin{array}{rlrl}
\dot{\mathbf{B}}+\nabla \times \mathbf{E} & =0, & & \nabla \times \mathbf{B}-\frac{1}{c_{0}^{2}} \dot{\mathbf{E}}=\mathbf{0}, \\
\nabla \cdot \mathbf{E} & =0, & \nabla \cdot \mathbf{B}=0 .
\end{array}
$$

\subsection{The Electromagnetic Potentials}

The electric scalar potential $\Phi$ and the magnetic vector potential $\mathbf{A}$ are introduced by the definitions

$$
\mathbf{E}=-\nabla \Phi-\dot{\mathbf{A}}, \quad \mathbf{B}=\nabla \times \mathbf{A}
$$

This definition satisfies the two homogeneous Maxwell equations in (7). The definition of $\boldsymbol{A}$ leaves its divergence $\boldsymbol{\nabla} \cdot \mathbf{A}$ arbitrary. We shall use the Lorentz gauge [13]

$$
\boldsymbol{\nabla} \cdot \mathbf{A}+\mu \epsilon \dot{\Phi}=0 .
$$

With this choice the two non-homogeneous Maxwell equations in terms of $\Phi$ and $\mathbf{A}$ separate into the wave equations

$$
\nabla^{2} \Phi-\mu \epsilon \bar{\Phi}=-\rho / \epsilon, \quad \nabla^{2} \mathbf{A}-\mu \epsilon \overline{\mathrm{A}}=-\mu \mathrm{j} .
$$




\section{THE ELECTROMAGNETIC FOUR-POTENTIAL}

Maxwell's equations can be presented in a compact manner* in the four-dimensional spacetime defined by the coondinates

$$
x_{1} \equiv x, \quad x_{2} \equiv y, \quad x_{3} \equiv z, \quad x_{4}=i c t
$$

where $x_{1}, x_{2}, x_{3}$ are spatial Cartesian coordinates, $i^{2}=-1$ is the imaginary unit, and $c=1 / \sqrt{\mu \varepsilon}$ is the speed of EM waves in the medium under consideration. In the sequel Roman subscripts will consistently go from 1 to 4 and the summation convention over repeated indices will be used unless otherwise stated.

\subsection{The Field Strength Tensor}

The unification can be expressed most conveniently in terms of the field-strength tensor F, which is a four-dimensional antisymmetric tensor constructed from the components of $\mathbf{E}$ and $\mathbf{B}$ as follows:

$$
\mathbf{F}=\left(\begin{array}{cccc}
0 & F_{12} & F_{13} & F_{14} \\
-F_{12} & 0 & F_{23} & F_{24} \\
-F_{13} & -F_{23} & 0 & F_{34} \\
-F_{14} & -F_{23} & -F_{34} & 0
\end{array}\right) \stackrel{\text { def }}{=} \beta\left(\begin{array}{cccc}
0 & c B_{3} & -c B_{2} & -i E_{1} \\
-c B_{3} & 0 & c B_{1} & -i E_{2} \\
c B_{2} & -c B_{1} & 0 & -i E_{3} \\
i E_{1} & i E_{2} & i E_{3} & 0
\end{array}\right)
$$

Here $\beta$ is an adjustment factor to be determined later. Similarly, introduce the four-current vector $\mathbf{J}$ as

$$
\mathbf{J}=\left\{\begin{array}{l}
J_{1} \\
J_{2} \\
J_{3} \\
J_{4}
\end{array}\right\} \stackrel{\text { def }}{=} \beta\left\{\begin{array}{l}
c \mu j_{1} \\
c \mu j_{2} \\
c \mu j_{3} \\
i \rho / \epsilon
\end{array}\right\}=\beta c\left\{\begin{array}{c}
\mu j_{1} \\
\mu j_{2} \\
\mu j_{3} \\
i \sqrt{\mu / \epsilon} \rho
\end{array}\right\} .
$$

Then, for arbitrary $\beta$, the non-homogeneous Maxwell equations, namely $\nabla \times \mathbf{B}-\mu \epsilon \dot{\mathbf{E}}=\mu \mathrm{j}$ and $\nabla \cdot \mathbf{E}=\rho / \epsilon$, may be presented in the compact "continuity" form $\dagger$

$$
\frac{\partial F_{i k}}{\partial x_{k}}=J_{i}
$$

The other two Maxwell equations, $\nabla \cdot \mathbf{B}=0$ and $\nabla \times \mathbf{E}+\dot{\mathbf{B}}=0$, can be presented as

$$
\frac{\partial F_{i k}}{\partial x_{m}}+\frac{\partial F_{m i}}{\partial x_{k}}+\frac{\partial F_{k m}}{\partial x_{i}}=0
$$

where the index triplet $(i, j, k)$ takes on the values $(1,2,3),(4,2,3),(4,3,1)$ and $(4,1,2)$.

* A form compatible with specjal relativity.

t The covariant form of these two equations. 7 


\subsection{The Four-Potential}

The EM "four-potential" $\phi$ is a four-vector whose components are constructed with the electric and magnetic potential components of $\boldsymbol{A}$ and $\boldsymbol{\Phi}$ :

$$
\phi=\beta\left\{\begin{array}{l}
\phi_{1} \\
\phi_{2} \\
\phi_{3} \\
\phi_{4}
\end{array}\right\} \stackrel{\text { deff }}{=}\left\{\begin{array}{c}
c A_{1} \\
c A_{2} \\
c A_{3} \\
i \Phi
\end{array}\right\} .
$$

It may then be verified that $F$ can be expressed as the four-curl of $\phi$, that is

$$
F_{i k}=\frac{\partial \phi_{k}}{\partial x_{i}}-\frac{\partial \phi_{i}}{\partial x_{k}}
$$

or in more detail and using commas to abbreviate partial derivatives:

$$
\mathbf{F}=\left(\begin{array}{cccc}
0 & \phi_{2,1}-\phi_{1,2} & \phi_{3,1}-\phi_{1,3} & \phi_{4,1}-\phi_{1,4} \\
\phi_{1,2}-\phi_{2,1} & 0 & \phi_{3,2}-\phi_{2,3} & \phi_{4,2}-\phi_{2,4} \\
\phi_{1,3}-\phi_{3,1} & \phi_{2,3}-\phi_{3,2} & 0 & \phi_{4,3}-\phi_{3,4} \\
\phi_{1,4}-\phi_{4,1} & \phi_{2,4}-\phi_{4,2} & \phi_{3,4}-\phi_{4,3} & 0
\end{array}\right) .
$$

\subsection{The Lagrangian}

With these definitions, the basic Lagrangian of electromagnetism can be stated as

$$
\begin{aligned}
L & =\frac{1}{4} F_{i k} F_{i k}-J_{i} \phi_{i}=\frac{1}{4} \beta^{2}\left(\frac{\partial \phi_{k}}{\partial x_{i}}-\frac{\partial \phi_{i}}{x_{k}}\right)^{2}-J_{i} \phi_{i} \\
& =\frac{1}{2} \beta^{2}\left(c^{2} B^{2}-E^{2}\right)-\frac{\beta^{2}}{\epsilon}\left(j_{1} A_{1}+j_{2} A_{2}+j_{3} A_{3}-\rho \Phi\right),
\end{aligned}
$$

in which

$$
B^{2}=\mathrm{B}^{T} \mathrm{~B}=B_{1}^{2}+B_{2}^{2}+B_{3}^{2}, \quad E^{2}=\mathrm{E}^{T} \mathrm{E}=E_{1}^{2}+E_{2}^{2}+E_{3}^{2} .
$$

Comparing the first term with the magnetic and electric energy densities $[2,19,20]$

$$
u_{m}=\frac{1}{2} \mathbf{B}^{T} \mathbf{H}=\frac{1}{2 \mu} B^{2}, \quad u_{e}=\frac{1}{2} \mathbf{D}^{T} \mathbf{E}=\frac{1}{2} \epsilon E^{2},
$$

we must have $\beta^{2} c^{2}=\beta^{2} /(\mu \epsilon)=1 / \mu$, from which

$$
\beta=\sqrt{\epsilon}
$$

\$ Lanczos [12] presents this Lagrangian for free space, but the expression (24) for an arbitrary material was found in none of the textbooks on electromagnetism listed in the References. 
Consequently the required Lagrangian is

$$
L=\frac{1}{2 \mu} B^{2}-\frac{1}{2} \epsilon E^{2}-\left(j_{1} A_{1}+j_{2} A_{2}+j_{3} A_{3}-\rho \Phi\right)
$$

The associated variational form is

$$
R=\int_{t_{0}}^{t_{1}} \int_{V} L d V d t
$$

where $V$ is the integration volume considered in the analysis. In theory $V$ extends over the whole space, but in the numerical simulation the integration is truncated at a distant boundary or special devices are used to treat the decay behavior at infinity.

\subsection{The Four-Field Eqrations}

On setting the variation of the functional (24) to zero we recover the field equations (1516). Taking the divergence of both sides of (15) and observing that $F$ is an antisymmetric tensor so that its divergence vanishes we get

$$
\frac{\partial J_{i}}{\partial x_{i}}=c \mu(\nabla \cdot j+\dot{\rho})=0,
$$

The vanishing term in parenthesis is the equation of continuity, which expresses the law of conservation of charge. The Lorentz gauge condition (10) may be stated as $\nabla \cdot \phi=0$. Finally, the potential wave equations (11) may be expressed in compact form as

$$
\phi_{i}=-J_{i}
$$

where $\square$ denotes the "four-wave-operator", also called the D'Alembertian:

$$
\stackrel{\text { def }}{=} \frac{\partial^{2}}{\partial x_{k} \partial x_{k}}=\frac{\partial^{2}}{\partial x_{1}^{2}}+\frac{\partial^{2}}{\partial x_{2}^{2}}+\frac{\partial^{2}}{\partial x_{3}^{2}}-\frac{\partial^{2}}{c^{2} \partial t^{2}}
$$

Hence each component of the four-potential $\phi$ satisfies an inhomogeneous wave equation. In free space, $J_{i}=0$ and each component satisfies the homogeneous wave equation. 


\section{THE AXISYMMETRIC TEST EXAMPLE}

The simplest example for testing the finite element formulation based on the four-potential variational principle is provided by the axisymmetric magnetic field generated by a uniform, steady current flowing through a straight, infinitely long conducting wire of circular cross section. In the present Section we derive expressions for the magnetostatic fields outside and within the conductor. These analytical solutions will be later compared with the finite element numerical solutions.

\subsection{The Free-Space Magnetic Field}

To take advantage of the axisymmetric geometry we choose a cylindrical coordinate system with the wire centerline as the longitudinal $z$-axis. The vector components in the cylindrical coordinate directions $r, \theta$ and $z$ are denoted by

$$
\begin{aligned}
& A_{1}, B_{1}, E_{1} \text { in the } r \text { direction } \\
& A_{2}, B_{2}, E_{2} \text { in the } \theta \text { direction } \\
& A_{3}, B_{3}, E_{3} \text { in the } z \text { direction }
\end{aligned}
$$

The electromagnetic fields will then vary in the radial direction $(r)$ but not in the angular $(\theta)$ and axial $(z)$ directions. Similarly, the current density that flows in the wire has only one nonzero component acting in the positive or negative $z$ direction; conventionally we select the positive direction.

In Cartesian coordinates the radial component of the electrostatic potential in free space can be calculated from the expression (see, e.g., $[2,10,18,19,20]$ )

$$
A_{z}=A_{3}=\frac{\mu_{0}}{4 \pi} \int_{V} \frac{j_{3}}{|\mathbf{r}|} d V
$$

where $|\mathbf{r}|$ is the distance between the elemental charge $j_{3} d V$ and the point in space at which we wish to find the field potential. The integral extends over the volume containing charges. This expression serves equally well in cylindrical coordinates. In fact, the transformation of $z$ components will be one to one if the center of the systems coincide.

As noted above the only non-vanishing component of the current vector is $j_{3} d S$ where $d S$ is the elemental cross sectional area of the conductor and $j_{3}$ is the current density in the $z$ direction. If $d \ell$ represents the differential length of the wire, then $\int_{S} j_{3} d V=\int_{S} j_{3} d S d \ell=$ $I d \ell=I d z$ and $|\mathbf{r}|=\sqrt{r^{2}+z^{2}}$. Substitution into Eq. (29) yields

$$
A_{3}(r)=\frac{\mu_{0} I}{4 \pi} \int_{-\infty}^{\infty} \frac{d z}{\sqrt{r^{2}+z^{2}}}
$$

This integral diverges, but this difficulty can be overcome by taking the wire to have a finite length $2 L$ symmetric with respect to the field point, that is large with respect to its diameter. Integrating between $-L$ and $+L$ we get

$$
A_{3}(r)=\frac{\mu_{0} I}{4 \pi} \int_{-L}^{L} \frac{d z}{\sqrt{r^{2}+z^{2}}}=\left.\frac{\mu_{0} I}{4 \pi} \ln \left(z+\sqrt{r^{2}+z^{2}}\right)\right|_{-L} ^{+L} .
$$


Expanding this equation in powers of $r / L$ and retaining only first-order terms gives

$$
A_{3}=-\left(\frac{\mu_{0} I}{2 \pi}\right) \ln r+C .
$$

where $C$ is an arbitrary constant. For subsequent developments it is convenient to select $C=\left(\mu_{0} I / 2 \pi\right) \ln R_{T}$, where $R_{T}$ is the "truncation radius" of the finite element mesh in the radial direction. Then

$$
A_{3}=-\left(\frac{\mu_{0} I}{2 \pi}\right) \ln \left(\frac{r}{R_{T}}\right) .
$$

With this normalization $A_{3}=0$ at $r=R_{T}$. Taking the curl of $\mathrm{A}$ gives the $\mathrm{B}$ field in cylindrical coordinates:

$$
\mathbf{B}=\nabla \times \mathbf{A}=\left\{\begin{array}{l}
B_{1} \\
B_{2} \\
B_{3}
\end{array}\right\}=\left\{\begin{array}{l}
B_{r} \\
B_{\theta} \\
B_{z}
\end{array}\right\}=\left\{\begin{array}{c}
\frac{1}{r} \frac{\partial A_{3}}{\partial \theta}-\frac{\partial A_{2}}{\partial z} \\
\frac{\partial A_{1}}{\partial z}-\frac{\partial A_{3}}{\partial r} \\
\frac{1}{r} \frac{\partial\left(r A_{2}\right)}{\partial r}-\frac{1}{r} \frac{\partial A_{1}}{\partial \theta}
\end{array}\right\}=\left\{\begin{array}{c}
0 \\
-\frac{\partial A_{3}}{\partial r} \\
0
\end{array}\right\} .
$$

It is seen that the only non-vanishing component of the magnetic flux density is

$$
B_{\theta} \equiv B_{2}=\mu_{0} H_{2}=-\frac{\partial A_{3}}{\partial r}=\frac{\mu_{0} I}{2 \pi r} .
$$

This expression is called the law of Biot-Savart in the EM literature.

\subsection{Magnetic Field Within the Conductor}

Again restricting our consideration to the static case, we have from Maxwell's equations in their integral flux form

$$
\oint_{C} \mathbf{H} \cdot d \mathbf{s}=\oint_{C} \mu^{-1} \mathbf{B} \cdot d \mathbf{s}=\int_{S} \mathbf{j} \cdot d \mathbf{S},
$$

where $C$ is a contour around the field point traversed counterclockwise with an oriented differential arclength $d s$ and $d S$ is the oriented surface element inside the contour. The term for the electric field disappears in this analysis because $\dot{\mathbf{E}}=\mathbf{0}$. From before we know that the right hand side of Eq. (35) is equal to the normal component of the current that Hows through the cross sectional area evaluated by the integral. In the free space case, this is the total current that flows through the conductor. But in the conductor the amount of current is a function of the distance $r$ from the center. Again using $I$ to represent the total current carried by the conductor, and $R$ the radius of the conductor, and assuming an uniform current density $j_{3}=I /\left(\pi R^{2}\right)$, the right hand side of (35) become_A

$$
\int_{S} \mathrm{j} \cdot d \mathrm{~S}=\int_{S} j_{3} d S=\frac{I}{\pi R^{2}} \int_{S} d S=I \frac{r}{R^{2}} .
$$


Evaluating the left hand side of the integral and solving for $B_{2}$ gives:

$$
2 \pi r \mu^{-1} B_{2}=I \frac{r^{2}}{R^{2}}, \quad B_{2}=\frac{\mu I r}{2 \pi R^{2}} .
$$

Comparing with (34) we see that if $\mu=\mu_{0}$ then $B_{2}$ is continuous at the wire surface $r=R$ and has the value $\mu_{0} I /(2 \pi R)$. But if $\mu \neq \mu_{0}$ there is a jump $\left(\mu-\mu_{0}\right) I /(2 \pi R)$ in $B_{2}$.

The magnetic potential $A_{3}$ within the conductor is easily computed by integrating $-B_{2}$ with respect to $r$ :

$$
A_{3}=-\frac{\mu I r^{2}}{4 \pi R^{2}}+C
$$

The value of $C$ is determined by matching (33) at $r=R$, since the potential must be continuous. The result can be written

$$
A_{3}=\frac{I}{2 \pi}\left[\frac{1}{2} \mu\left(1-\frac{r^{2}}{R^{2}}\right)-\mu_{0} \ln \left(\frac{R}{R_{T}}\right)\right] .
$$

The preceding expressions (33)-(40) for $A_{3}$ could also be derived in a somewhat more direct fashion by integrating the ordinary differential equation $\nabla^{2} A_{3}=r^{-1}\left(\partial\left(r \partial A_{3} / \partial r\right) \partial r\right)=$ $\mu j_{3}$ to which the second of (11) reduces.

\section{FINITE ELEMENT DISCRETIZATION}

\subsection{The Lagrangian in Cylindrical Coordinates}

To construct finite element approximations we need to express the Lagrangian (24)

$$
L=\frac{1}{2 \mu} B^{2}-\frac{1}{2} \epsilon E^{2}-\left(j^{T} \mathrm{~A}-\rho \Phi\right),
$$

in terms of the potentials written in cylindrical coordinates. For $B^{2}$ we can use the expression of the curl (33)

$$
B^{2}=\left(\frac{1}{r} \frac{\partial A_{3}}{\partial \theta}-\frac{\partial A_{2}}{\partial z}\right)^{2}+\left(\frac{\partial A_{1}}{\partial z}-\frac{\partial A_{3}}{\partial r}\right)^{2}+\left(\frac{1}{r} \frac{\partial\left(r A_{2}\right)}{\partial r}-\frac{1}{r} \frac{\partial A_{1}}{\partial \theta}\right)^{2},
$$

For $E^{2}$ we need the cylindrical-coordinate gradient formulas

$$
\mathbf{E}=\left\{\begin{array}{c}
E_{1} \\
E_{2} \\
E_{3}
\end{array}\right\}=\left\{\begin{array}{c}
E_{r} \\
E_{\theta} \\
E_{z}
\end{array}\right\}=-\left\{\begin{array}{c}
\frac{\partial \Phi}{\partial r}+\dot{A}_{1} \\
\frac{1}{r} \frac{\partial \Phi}{\partial \theta}+\dot{A}_{2} \\
\frac{\partial \Phi}{\partial z}+\dot{A}_{3}
\end{array}\right\}
$$

so that

$$
E^{2}=\mathbf{E}^{T} \mathbf{E}=\left(\frac{\partial \Phi}{\partial r}+\frac{\partial A_{1}}{\partial t}\right)^{2}+\left(\frac{1}{r} \frac{\partial \Phi}{\partial \theta}+\frac{\partial A_{2}}{\partial t}\right)^{2}+\left(\frac{\partial \Phi}{\partial z}+\frac{\partial A_{3}}{\partial t}\right)^{2}
$$


In the axisymmetric case, $A_{1}=A_{2}=0$; furthermore $A_{z}=A_{3}$ is only a function of the radial distance from the wire. Therefore $\partial A_{3} / \partial \theta=\partial A_{3} / \partial z=0$. From symmetry considerations we also know that the electric field cannot vary in the $\theta$ and $z$ directions, which gives $\partial \Phi / \partial z=\partial \Phi / \partial \theta=0$. Finally, the only nonvanishing current density component is $j_{3}$. Consequently the Lagrangian (41) simplifies to

$$
L=\frac{1}{2 \mu}\left(\frac{\partial A_{3}}{\partial r}\right)^{2}-\frac{1}{2} \epsilon\left[\left(\frac{\partial \Phi}{\partial r}\right)^{2}+\left(\frac{\partial A_{3}}{\partial t}\right)^{2}\right]-\left(j_{3} A_{3}-\rho \Phi\right) .
$$

\subsection{Constructing EM Finite Elements}

To deal with this particular axisymmetric problem a two-node "line" finite element extending in the radial $r$ direction is sufficient. In the following we deal with an individual element identified by superscript $e$. The two element end nodes are denoted by $i$ and $j$. The electric potential and the magnetic potential $A_{3} \equiv A_{z}$ are interpolated over each element as

$$
\Phi^{e}=\mathbf{N}_{\Phi}^{e} \Phi^{e}, \quad A_{3}^{e}=\mathbf{N}_{A}^{e} A_{3}^{e},
$$

Here row vectors $N_{\Phi}^{e}$ and $N_{A}^{e}$ contain the finite element shape functions for $\Phi^{e}$ and $A_{3}^{e}$, respectively, which are only functions of the radial coordinate $r$ :

$$
N_{\Phi}^{e}=\left\langle\begin{array}{lll}
N_{\Phi i}^{e}(r) & N_{\Phi j}^{e}(r)
\end{array}\right), \quad N_{A}^{e}=\left\langle N_{A i}^{e}(r) \quad N_{A j}^{e}(r)\right\rangle
$$

and column vectors $\Phi^{e}$ and $A_{3}^{e}$ contain the nodal values of $\Phi$ and $A_{3}$, respectively, which are only functions of time $t$ :

$$
\Phi^{e}=\left\{\begin{array}{c}
\Phi_{i}(t) \\
\Phi_{j}(t)
\end{array}\right\}, \quad \mathbf{A}_{3}^{e}=\left\{\begin{array}{c}
A_{3 i}(t) \\
A_{3 j}(t)
\end{array}\right\}
$$

Substitution of these finite element assumptions into the Lagrangian (45) and then into Eq. (25) yields the variational integral as sum of element contributions $R=\sum_{e} R^{e}$, where

$$
\begin{aligned}
R^{e}=\int_{t_{0}}^{t_{1}} \int_{V}= & \frac{1}{2 \mu}\left(\frac{\partial \mathrm{N}_{A}^{e}}{\partial r} \mathbf{A}_{3}^{e}\right)^{2}-\frac{1}{2} \epsilon\left[\left(\frac{\partial N_{\Phi}^{e}}{\partial \tau} \Phi^{e}\right)^{2}+\left(\frac{\partial N_{A}^{e}}{\partial t} \dot{A}_{3}^{e}\right)^{2}\right] \\
& -\left(j N_{A}^{e} A_{3}^{e}-\rho N_{\Phi}^{e} \Phi^{e}\right) d V^{e} d t .
\end{aligned}
$$

where $V^{e}$ denotes the rolume of the element. Taking the variation with respect to the element node values gives

$$
\begin{aligned}
\delta R^{e} & =\int_{t_{0}}^{t_{1}} \int_{V^{e}}\left(\delta \mathbf{A}_{3}^{e}\right)^{T}\left[\frac{1}{\mu}\left(\frac{\partial N_{A}^{e}}{\partial r}\right)^{T} \frac{\partial N_{A}^{e}}{\partial r} A_{3}^{e}+\epsilon\left(N_{A}^{e}\right)^{T} N_{A}^{e} \bar{A}_{3}^{e}-j_{3}\left(N_{A}^{e}\right)^{T}\right] \\
& +\int_{t_{0}}^{t_{1}} \int_{V^{e}}\left(\delta \Phi^{e}\right)^{T}\left[-\epsilon\left(\frac{\partial N_{\Phi}^{e}}{\partial r}\right)^{T} \frac{\partial N_{\Phi}^{e}}{\partial r} \Phi^{e}+\rho\left(N_{\Phi}^{e}\right)^{T}\right] d V^{e} d t .
\end{aligned}
$$


On applying fixed-end initial conditions at $t=t_{0}$ and $t=t_{1}$ and the lemma of the calculus of variations, we proceed to equate each of the expressions in brackets to zero. From the first bracket we obtain for each element the following second-order dynamic equations for the magnetic potential at the nodes, which are purposedly written in a notation resembling the mass-stiffness-force equations of mechanics:

$$
M_{A}^{e} \bar{A}_{3}^{e}+K_{A}^{e} A_{3}^{e}=f_{A}^{e},
$$

where

$$
\begin{gathered}
\mathbf{M}_{A}^{e}=\int_{V^{\bullet}} \epsilon\left(\mathrm{N}_{A}^{e}\right)^{T} \mathrm{~N}_{A}^{e} d V^{e}, \quad \mathbf{K}_{A}^{e}=\int_{V^{\cdot}} \frac{1}{\mu}\left(\frac{\partial \mathrm{N}_{A}^{e}}{\partial r}\right)^{T} \frac{\partial \mathrm{N}_{A}^{e}}{\partial r} d V^{e}, \\
\mathrm{f}_{A}^{e}=\int_{V^{*}} j_{3}\left(\mathrm{~N}_{A}^{e}\right)^{T} d V^{e} .
\end{gathered}
$$

From the second bracket we obtain for the electric potential a simpler relation which does not involve time derivatives, i.e, is static in nature:

$$
\mathbf{K}_{\Phi}^{e} \Phi^{e}=\mathbf{f}_{\Phi}^{e}
$$

where

$$
\mathbf{K}_{\Phi}^{e}=\int_{V^{\cdot}} \epsilon\left(\frac{\partial \mathbf{N}_{\Phi}^{e}}{\partial r}\right)^{T} \frac{\partial \mathbf{N}_{\Phi}^{e}}{\partial r} d V^{e}, \quad \mathbf{f}_{\Phi}^{e}=\int_{V^{\mathcal{E}}} \rho\left(\mathbf{N}_{\Phi}^{e}\right)^{T} d V^{e} .
$$

Assembling these equations in the usual way we obtain the semidiscrete master finite element equations:

$$
\begin{aligned}
\mathbf{M}_{A} \overline{\mathbf{A}}_{3}+\mathbf{K}_{A} \mathbf{A}_{3} & =\mathbf{f}_{A}, \\
\mathbf{K}_{\Phi} \Phi & =\mathbf{f}_{\Phi} .
\end{aligned}
$$

\subsection{The Static Case}

In time-independent problems, the term $\overline{\mathrm{A}}_{3}$ disappears from (56) and the master finite element equations of electromagnetostatics become

$$
K_{A} A_{3}=f_{A}, \quad K_{\Phi} \Phi=f_{\Phi} .
$$

If the current density and charge distributions are known a priori then these two equations may be solved separately. If only the charge distribution $\rho$ is known then the second equation should be solved first to obtain the electric field $\mathbf{E}$ as gradient of the computed electric potential $\Phi$; then the current density $j$ can be obtained from Ohm's law (6) and used to computed the force vector $f_{A}$ of the first equation, which is then solved for the magnetic potential. Conversely, if only the current density distribution is known a priori the preceding steps are reversed.

For the present test problem the current distribution is assumed to be known, and we shall be content with solving the first equation for the magnetic flux. 


\section{6.f An Alternative Semidiscretization}

If upon setting the brackets of the variation (50) to zero we multiply them through by $\mu$ and $1 / \epsilon$, respectively, the expressions for the mass, stiffness and force matrices become

$$
\begin{aligned}
& M_{A}^{e}=\int_{V \cdot} \frac{1}{c^{2}}\left(N_{A}^{e}\right)^{T} \cdot N_{A}^{e} d V^{e}, \mathbf{K}_{A}^{e}=\int_{V^{e}}\left(\frac{\partial N_{A}^{e}}{\partial r}\right)^{T} \frac{\partial N_{A}^{e}}{\partial r} d V^{e}, f_{A}^{e}=\int_{V \cdot} \mu j_{3} N_{A}^{e}{ }^{T} d V^{e}, \\
& \mathbf{K}_{\phi}^{e}=\int_{V \cdot}\left(\frac{\partial N_{\phi}^{e}}{\partial r}\right)^{T} \frac{\partial N_{\phi}^{e}}{\partial r} d V, \quad \mathbf{f}_{\Phi}^{e}=\int_{V \cdot} \frac{1}{\epsilon} \rho\left(N_{\phi}^{e}\right)^{T} d V .
\end{aligned}
$$

The matrices $\mathbf{M}$ and $\mathbf{K}$ above are quite similar to the capacitance and reactance matrices, respectively, obtained in the potential analysis of acoustic fluids $[7,8]$. Another attractive feature of (58) is that $\mathbf{K}_{A}=\mathbf{K}_{\phi}$ if the shape functions of both potentials coalesce, as is natural to assume. These advantages are, however, more than counterbalanced by the fact that "jump forces" contributions to $f_{A}$ and $f_{\Phi}$ arise on material interfaces where $\mu$ and $\epsilon$ change abruptly, and the proper handling of such forces substantially complicates the programming logic. Note that this issue does not arise in the treatment of homogeneous acoustic fluids.

\subsection{Applying Boundary Conditions}

The finite element mesh is necessarily terminated at a finite size, which for the test problem is defined as the truncation radius $R_{T}$ alluded to in Section 5.1. In static calculations the material outside the FE mesh may be viewed as having zero permeability $\mu$, or, equivalently, infinite stiffness or zero potential. It follows that the potential value at the node located on the truncation radius may be prescribed to be zero. This is the only essential boundary condition necessary for this particular problem.

\section{NUMERICAL VALIDATION}

\subsection{Finite Element Model}

The test problem consists of a wire conductor of radius $R$ transporting a unit current density. For this problem the finite element mesh is completely defined if we specify the radial node coordinates $r_{i}^{e}=r_{n}^{e}$ and $r_{j}^{e}=r_{n+1}^{e}$ for each element $e$. If the mesh contains $N_{e c}$ elements inside the conductor, those elements are numbered $e=1,2, \ldots N_{e c}$ and nodes $n=1,2, \ldots N_{e c}+1$ starting from the conductor center outwards. The first node $(n=1)$ is at the conductor center $r=0$ and node $n=N_{e c}+1$ is placed at the conductor boundary $r=R$. The mesh is then continued with $N_{\text {ef }}$ elements into free space, with a double node at the counductor boundary. The last node is placed at $r=R_{T}$ at which point the free space mesh is truncated; usually $R_{T}=4 R$ to $5 R$. Although the mesh appears to be one-dimensional, a typical element actually forms a "tube" of longitudinal axis $z$, internal radius $r_{i}^{e}$ and external radius $r_{j}^{e}$, extending a unit distance along $z$. 


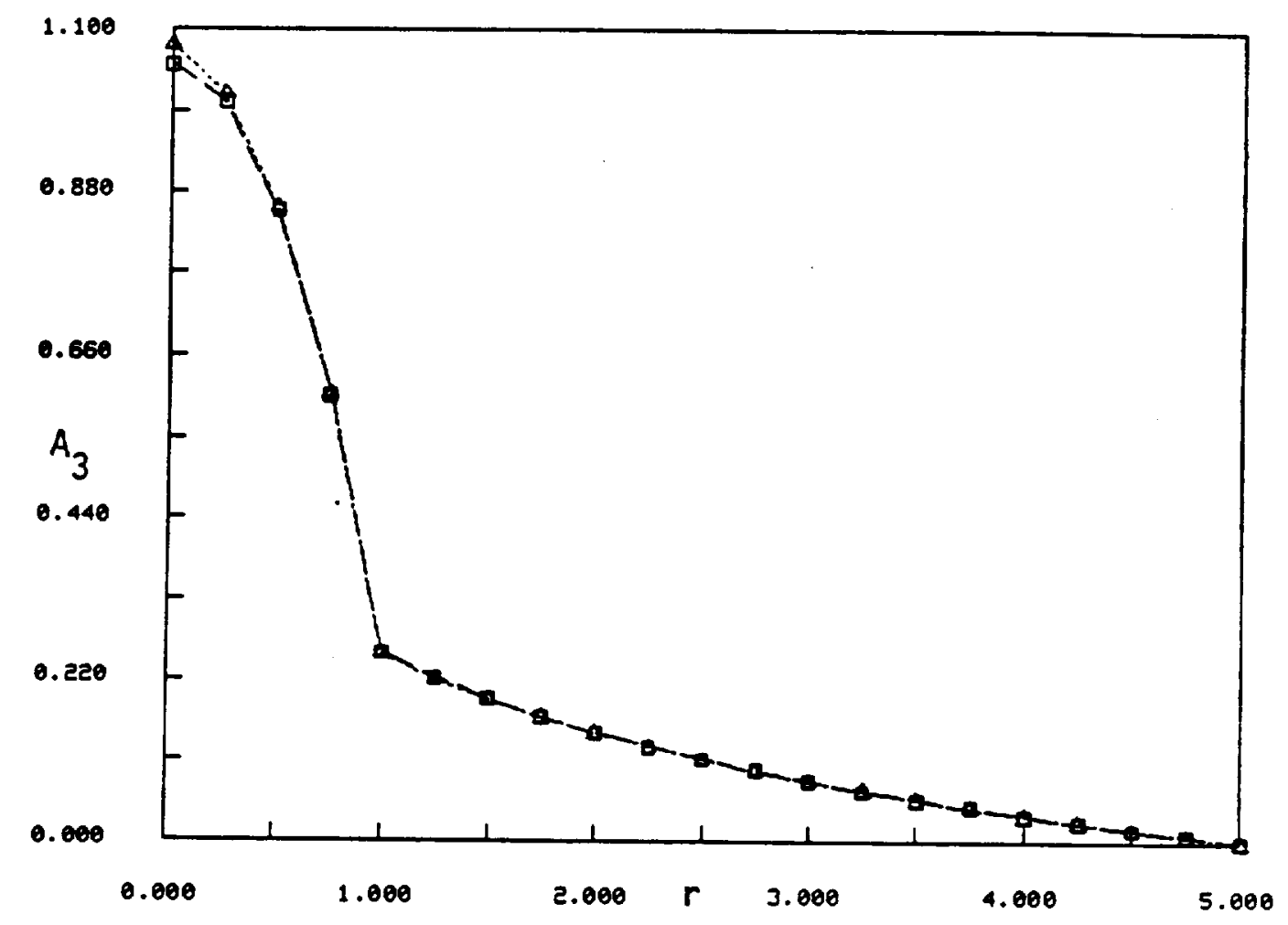

Figure 1. Magnetic potential As vs. distance from center $r$, $\mu_{\text {wirs }}=10.0$ : finite element values (triangles) and analytical values (squares).

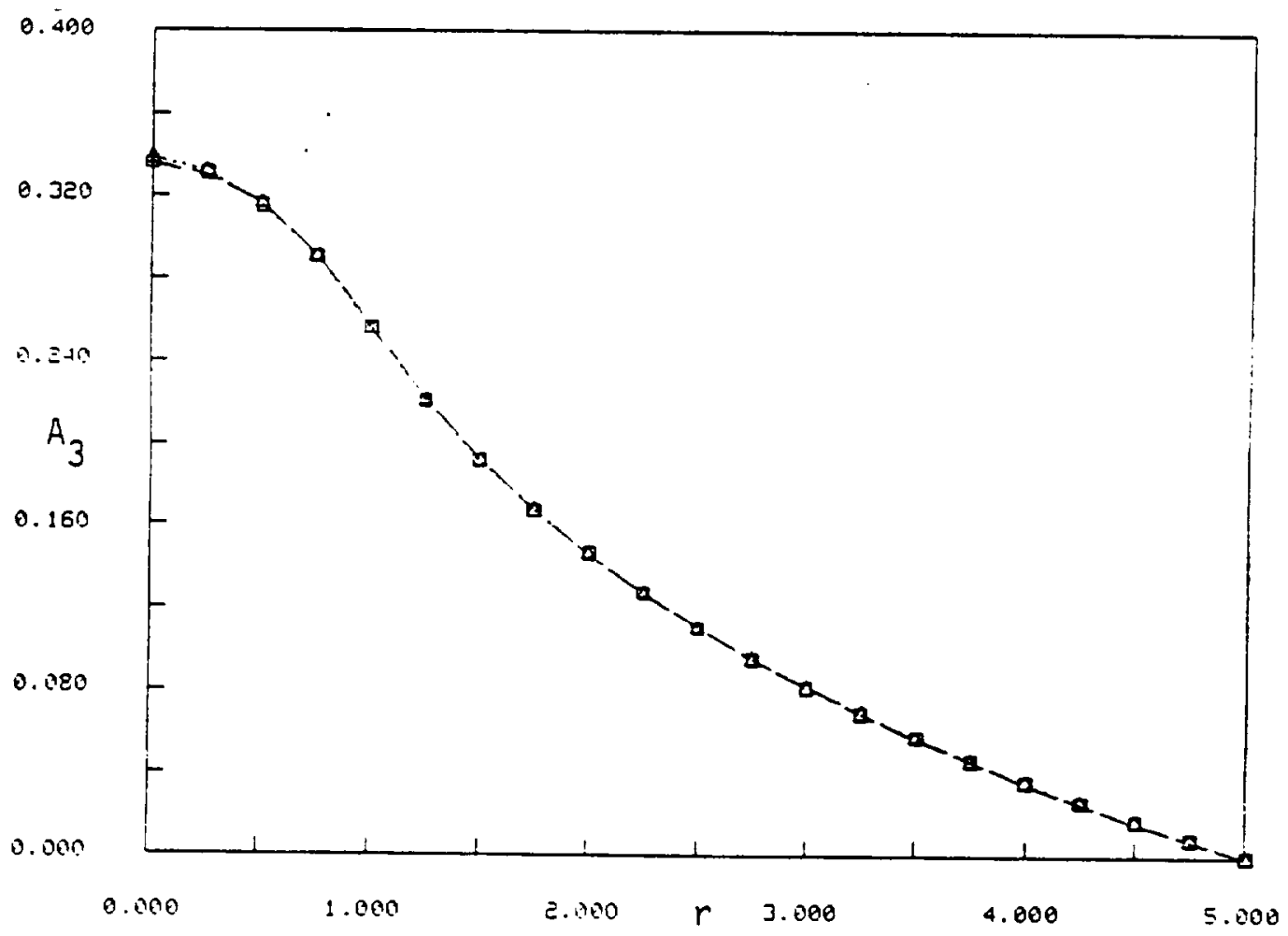

Figure 2. Magnetic potential $A_{3}$ vs. distance from center $r$, twire $_{1}=1.0$ : finite element values (triangles) and analytical values (squares). 


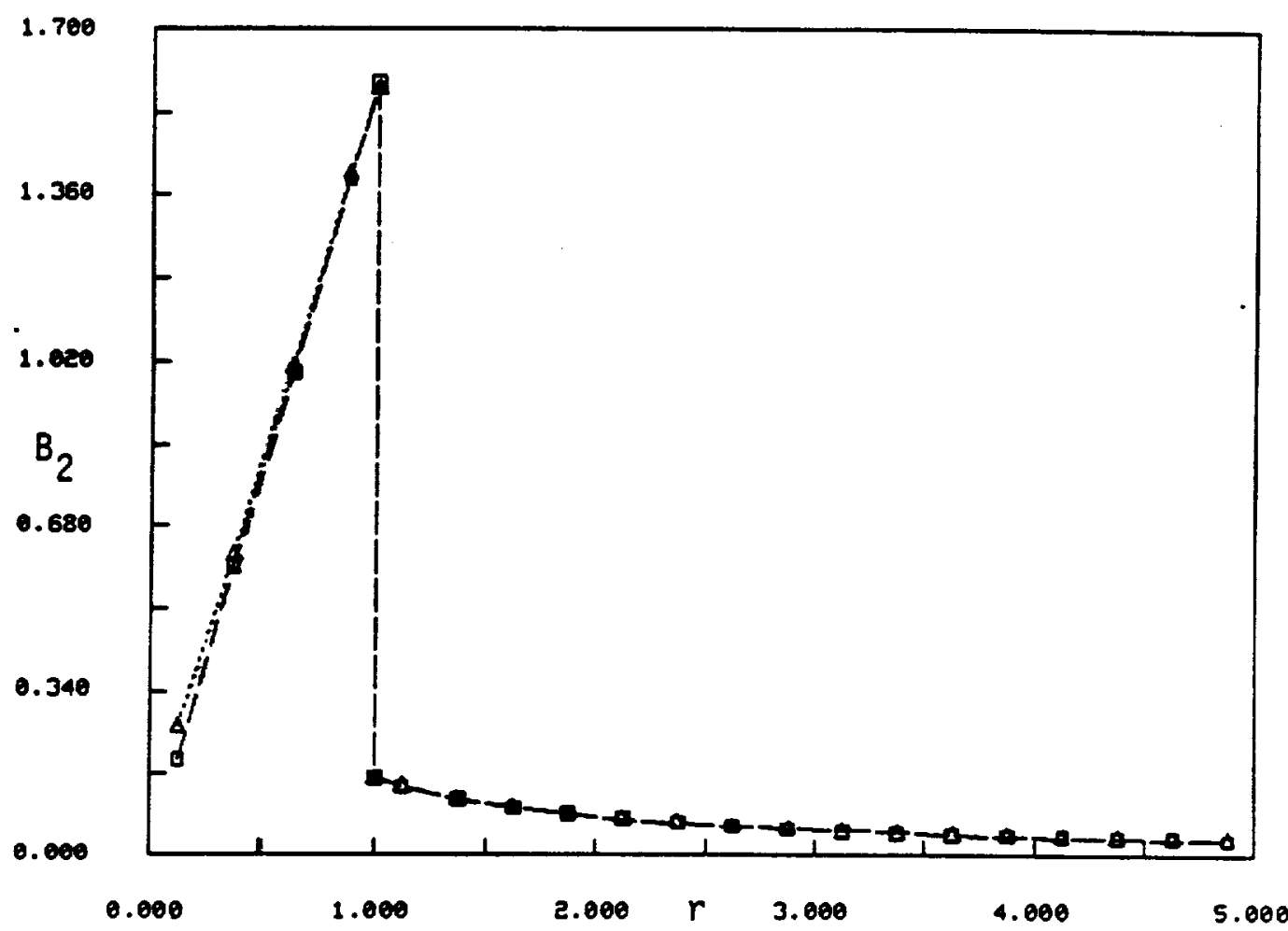

Figure 3. Magnetic flux density $B_{2}$ vs. distance from center $r, \mu_{\text {wire }}=10.0$ : finite element values (triangles) and analytical values (squares). Values shown on the interface $r=1$ with dark symbols have been extrapolated from element center values to display the jump more accurately; this extrapolation scheme has not been used elsewhere.

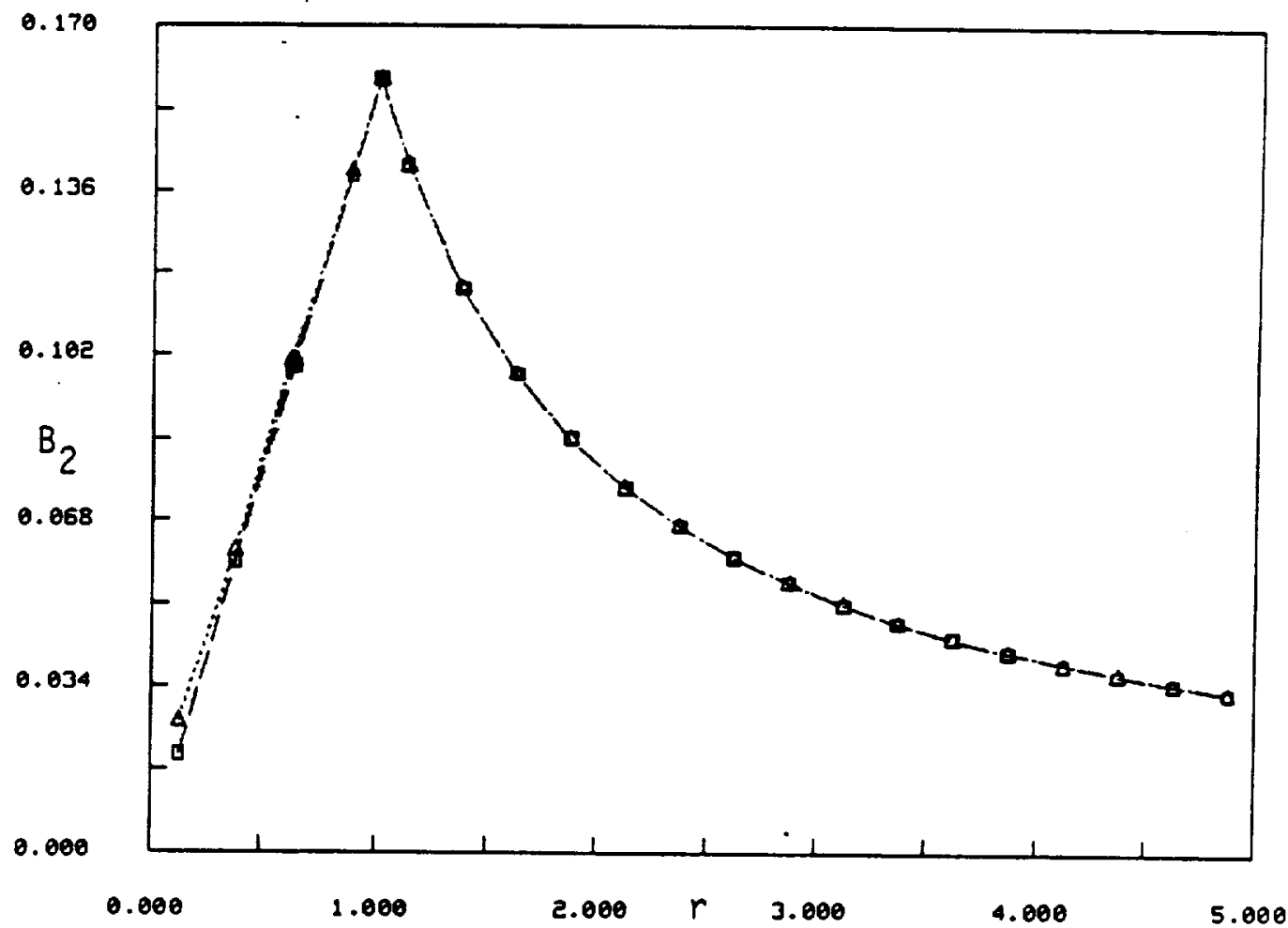

Figure 4. Magnetic $\theta u x$ density $B_{2}$ vs. distance from center $r, \mu_{w i r e}=1.0$ : finite element values (triangles) and analytical values (squares). 
For the present study the magnetic potential was linearly interpolated in $r$, using the linear shape functions

$$
N_{A}^{e}=\left\langle\frac{1}{2}(1-\xi) \quad \frac{1}{2}(1+\xi)\right\rangle,
$$

where $\xi$ is the dimensionless isoparametric coordinate that varies from -1 at node $i$ to +1 at node $j$. This interpolation provides for $C^{0}$ continuity of the potential inside the conductor and in free space.

For the calculation of the element stiffnesses and force vectors, it was assumed that the permeability $\mu$ and the current density $j_{3}$ were uniform over the element. Then analytical integration over the element geometry gives

$$
\mathbf{K}_{A}^{e}=\frac{\mu r_{m}}{\ell}\left[\begin{array}{rr}
1 & -1 \\
-1 & 1
\end{array}\right], \quad \mathbf{f}_{A}^{e}=j_{3} \ell\left\{\begin{array}{l}
\frac{1}{6}\left(2 r_{i}^{e}+r_{j}^{e}\right) \\
\frac{1}{6}\left(r_{i}^{e}+2 r_{j}^{e}\right)
\end{array}\right\}
$$

where $r_{m}=\frac{1}{2}\left(r_{i}^{e}+r_{j}^{e}\right)$ is the mean radius and $\ell=r_{j}^{e}-r_{i}^{e}$ the radial length. For the test problem, $\mu$ is constant inside the conductor whereas outside it $\mu=\mu_{0}$ was assumed to be unity. The longitudinal current density is $j_{3}=I /\left(\pi R^{2}\right)$ inside the conductor whereas outside it $j_{3}$ vanishes.

The master stiffness matrix and force vector were assembled following standard finite element techniques. The only essential boundary condition was the setting of the nodal potential on the truncation boundary to zero, as explained in Section 6.5. The modified master equations were processed by a conventional symmetric skyline solver, which provided the value of the magnetic potential at the mesh nodes. The magnetic flux density $B_{2}$, which is constant over each element, was recovered in element by element fashion through the simple finite difference scheme

$$
B_{2}^{e}=-\frac{\partial A_{3}}{\partial r} \approx \frac{A_{3 i}^{e}-A_{3 j}^{e}}{\ell}
$$

This value is assigned to the center of element $e$.

\subsection{Numerical Results}

The numerical results shown in Figures 1 through 6 pertain to a unit radius conductor ( $R=1$ ), with the external (free space) mesh truncated at $R_{T}=5$. The element radial lengths $r_{j}^{e}-r_{i}^{e}$ were kept constant and equal to 0.25 , which corresponds to 4 internal and 16 external elements.

The computed values of the potential $A_{3}$ are compared with the analytical solution given by Eqs. (33) and (40). As can be seen the agreement is excellent. The comparison between computed and analytical values of the magnetic flux density $B_{2}$ shows excellent agreement except for the last element near the wire center, at which point the difference scheme (61) loses accuracy. The permeability of free space is conventionally selected to be unity. Figures 1,3 , and 5 illustrate the case where the wire permeability $\mu_{w i r e}$ is set to 10.0 , whereas Figures 2, 4, and 6 are for the case in which $\mu_{\text {wire }}$ is 1.0 , that is, same as in free space. (The value of the susceptibility $\epsilon$ does not appear in these magnetostatic 


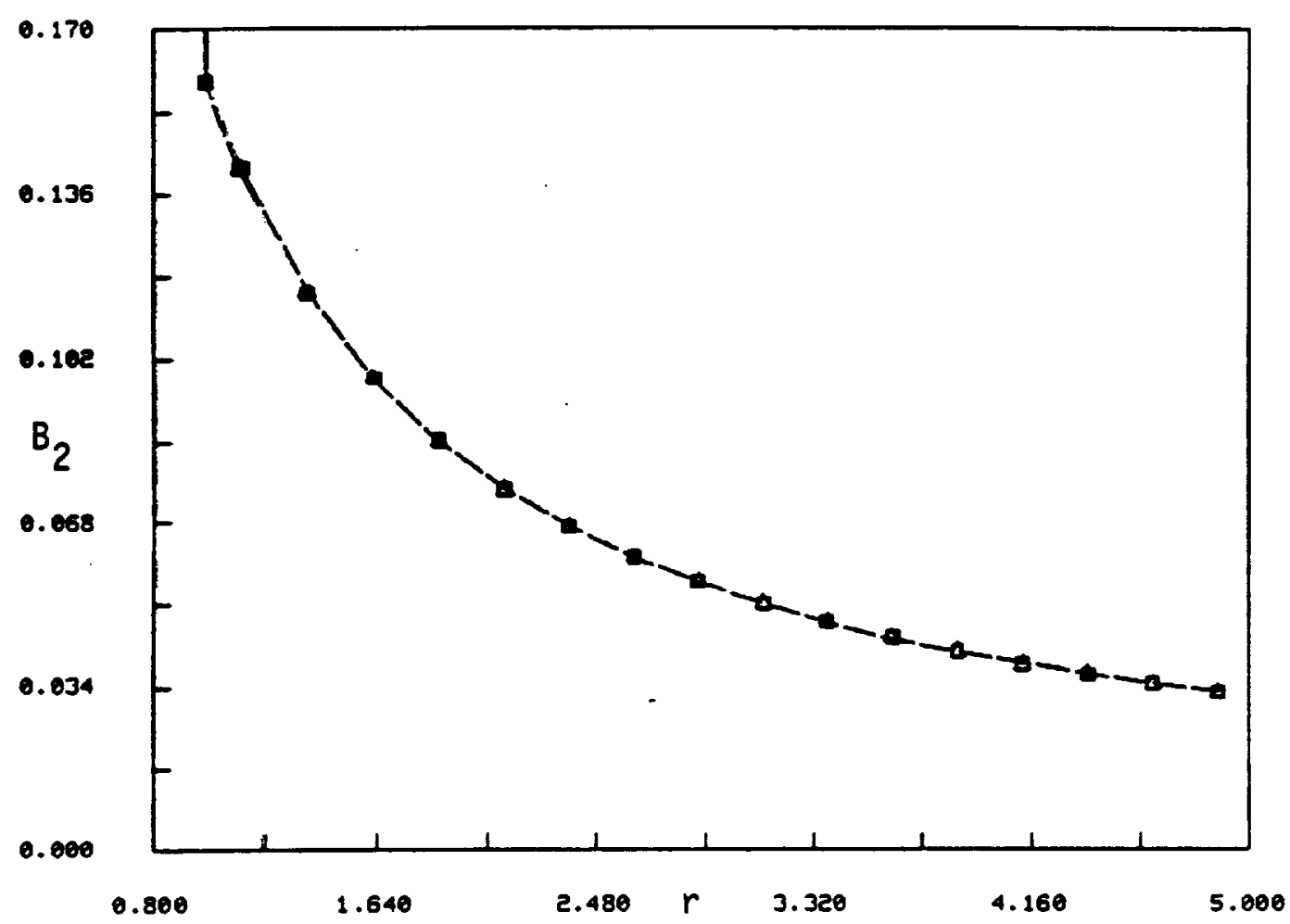

Figure 5. Restriction of Figure 3 to $r>R=1$, $\mu_{\text {wire }}=10.0$, showing free space magnetic flux density in more detail.

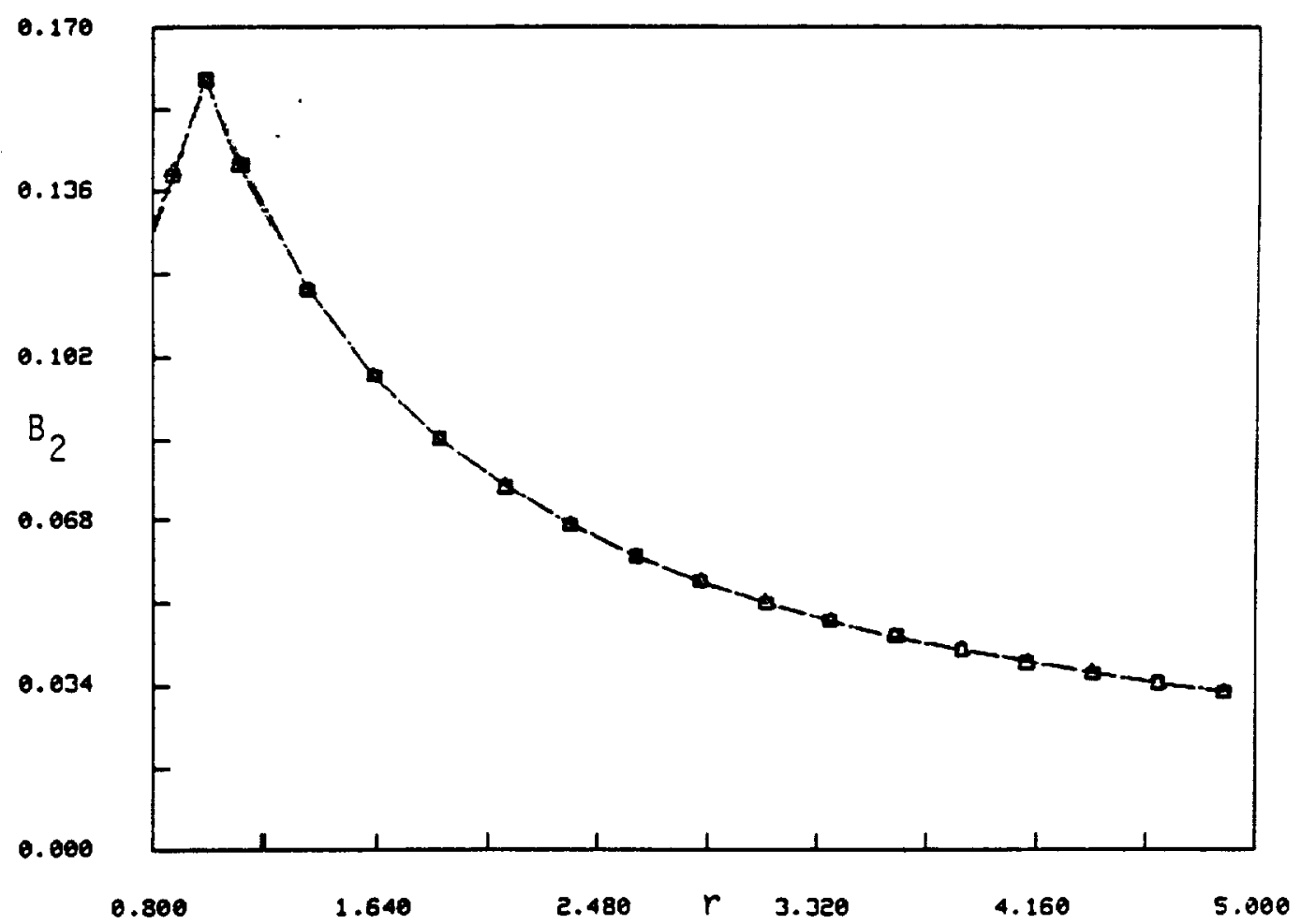

Figure 6. Restriction of Figure 4 to $r>R=1, \mu_{\text {wire }}=1.0$, showing free space magnetic flux density in more detail. 
computations.) Figures 1 and 2 show computed and analytical magnetic potentials. The slope discontinuity at $r=1$ in Figure 1 is a consequence of the change in permeability $\mu$ from the wire material to free space. Figures 3 and 4 show the computed and analytical magnetic flux densities. As discussed in Section 5.2, the jump at $r=1$ in Figure 3 is due to the change in permeability $\mu$ from the material to free space. Figures 5 and 6 show the computed and analytical magnetic flux densities in free space with more detail. Note that Figures 5 and 6 for $r>1$ are identical; this is the expected result because, as shown in Section 5.1, the free-space magnetic flux field depends only upon the current enclosed by a surface integral around the wire and not on the details of the interior field distribution.

In summary, the finite element model performed very accurately in the test problem and converged, as expected, to the analytical solution as the size of the elements decreased.

\section{CONCLUSIONS}

The results obtained in the one-dimensional steady-state case are encouraging, and appear to be extensible to two- and three-dimensional problems without major difficulties. The electric field remains effectively decoupled from the magnetic field except through Ohm's law. Care must be taken, however, in modeling the forcing function terms so as to avoid the appearance of discontinuity-induced forces at physical interfaces.

The next step in achieving the goal of a finite element model for a superconductor field is to study the time-dependent case, starting with harmonic currents and proceeding eventually to general transients. The code for this is currently written, but a suitable analytical solution for comparison with computed responses is still being developed.

If encouraging results are obtained in the dynamic case, thermocoupling effects will be added to the code. References $[3,17,22]$ discuss several different approaches applicable to various contexts (e.g. eddy currents) and these will have to be investigated for suitability for capturing the couplings effects that are relevant to the superconducting problem.

After modeling the coupling effects, the next step will be to model the superconducting fields. The feasibility of using the current model for superconductor applications is great, as the current density of a superconductor can be approximated by the standard current density multiplied by a constant squared. This constant is called the London penetration depth. Other analytical models that possess similar characteristics have been developed and are presented in Ref. [11]. 


\section{Acknowledgements}

This work was supported by NASA Lewis Research Center under Grant NAG 3-934, monitoreḍ by Dr. C. C. Chamis.

\section{RPTPRDNCDS}

1. Davies, J. B., The Finite Element Method, Chapter 2 in Numerical Techniques for Microwave and Millimeter-Wave Pasive Structures, T. Itoh (ed.), Wiley, New York, 1989

2. Eyges, L. The Clascical Electromagnetic Field, Dover, New York, 1980

3. Fano, RM., Chn, L.J., and Adler, R.B., Electromagnetic Fields, Energy, and Forces, John Wiley and Sons, Inc., New York, 1960

4. Felippa, C. A. and Geers, T. L., Partitioned Analysis of Coupled Mechanical Systems, Engineering Computatione, 5,1988 , pp. 123-133

5. Felippa, C. A., The Extended Free Formulation of Finite Elements in Linear Elasticity, Journal of Applied Mechanics, 56, 3, 1989, pp. 609-616

6. Folippa, C. A. and Militello, C., The Variational Formulation of High-Performance Finite Elements: Parametrized Variational Principles, (with C. Militello), submitted to Computers Structures, 1989

7. Felippa, C. A. and Militello, C., Developments in Variational Methods for High-Performance Plate and Shell Elemenits, to be presented at the ASME Winter Annual Meeting, San Francisco, December $1989^{\circ}$

8. Felippa, C. A. and Ohayon, R. Treatment of Coupled Fluid-Structure Interaction Problems by a Mixed Variational Principle, Proceedings 7 th International Conference on Finite Element Methods in Fluids, ed. by T.J. Chung et.al., University of Alabama Press, Huntsville, Alabama, April 1989, pp. 555-563

9. Felippa, C. A. and Ohayon, R. Mixed Variational Formulation of Finite Element Analysis of Acousto-elastic Fluid-Structure Interaction, submitted to Journal of Fluids $\&$ Structures, 1989

10. Grant, I.S., and Phillips, W.R., Electromagnetism, John Wiley and Sons, Inc., New York, 1975

11. Kittel, C., Introduction to Solid State Physics, 6th. ed, John Wiley and Sons, Inc., New York, 1986

12. Lanczos, C. The Variational Principles of Mechanics, Univ. of Toronto Press, Toronto, 1949

13. Lorentz, H. A., Theory of Electrons, 2nd. ed, Dover, New York, 1952

14. Militello, C. and Felippa, C. A., A Variational Justification of the Assumed Natural Strain Formulation of Finite Elements: I. Variational Principles, (with C. Militello), submitted to Computers \& Structures, 1988 
15. Militello, C. and Felippa, C. A., A Variational Justification of the Assumed Natural Strain Formulation of Finite Elements: II. The $C^{0}$-Node Plate Element, submitted to Computers \& Structures, 1988

16. Park, K. C. and Felippa, C. A., Partitioned Analysis of Coupled Systems, Chapter 3 in Computational Methods for Transient Analysis, T. Belytachko and T. J. R. Hughes, eds., North-Holland, Amsterdam-New York, 1983

17. Parkus, H., ed., Electromagnetic Interactions in Elastic Solids, Springer-Verlag, Berlin, 1979

18. Purcell, E.M., Electricity and Magnetism, Vol. 2, McGraw-Hill, New York, 1985

19. Rojanski, V., The Electromagnetic Field, Dover, New York, 1979

20. Shadowitz, A. The Electromagnetic Field, Dover, New York, 1975

21. Trowbridge, C. W., Numerical Solution of Electromagnetic Field Problems in Two and Three Dimensions, Chapter 18 in Numerical Methods in Coupled Problems, ed. by R. Lewis et.ad, Wiley, London, 1984

22. Yuan, K.-Y., Moon, F. C. and Abel, J. F., Elastic Conducting Structures in Pulsed Magnetic Fields, Chapter 19 in Numerical Methods in Coupled Problems, ed. by R. Lewis et.al., Wiley, London, 1984 


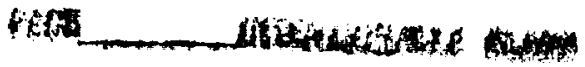




\section{APPENDIX: COMPUTER PROGRAM}

This Appendix lists the computer program used to teat the new electromagnetic elements on the axisymmetric test example. Sections of the program that pertain to the in-core skyline solver 8KYFAC/SKYSOL and the command language reader TinjCIip are not listed here. Their sonrce code is presented in the following pablications:

Felippa, C. A., Solution of Equations with Skyline-Stored Symmetric Coefficient Matrix, Computers \& Structures, 6, 1975, pp. 13-25

Felippa, C. A., A Command Reader for Interactive Programming, Engineering Computations, 2, No. 3, 1985, pp. 203-238

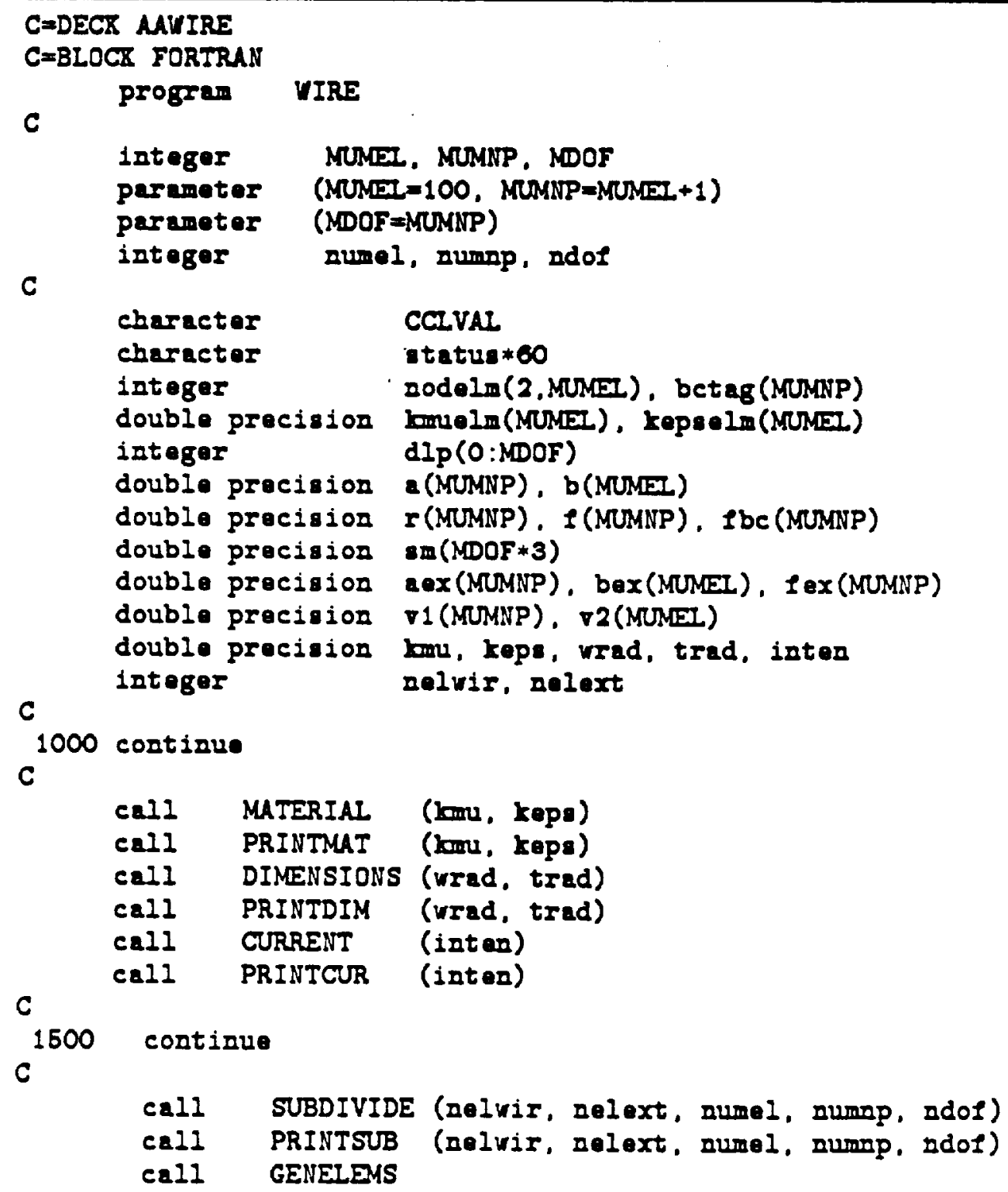




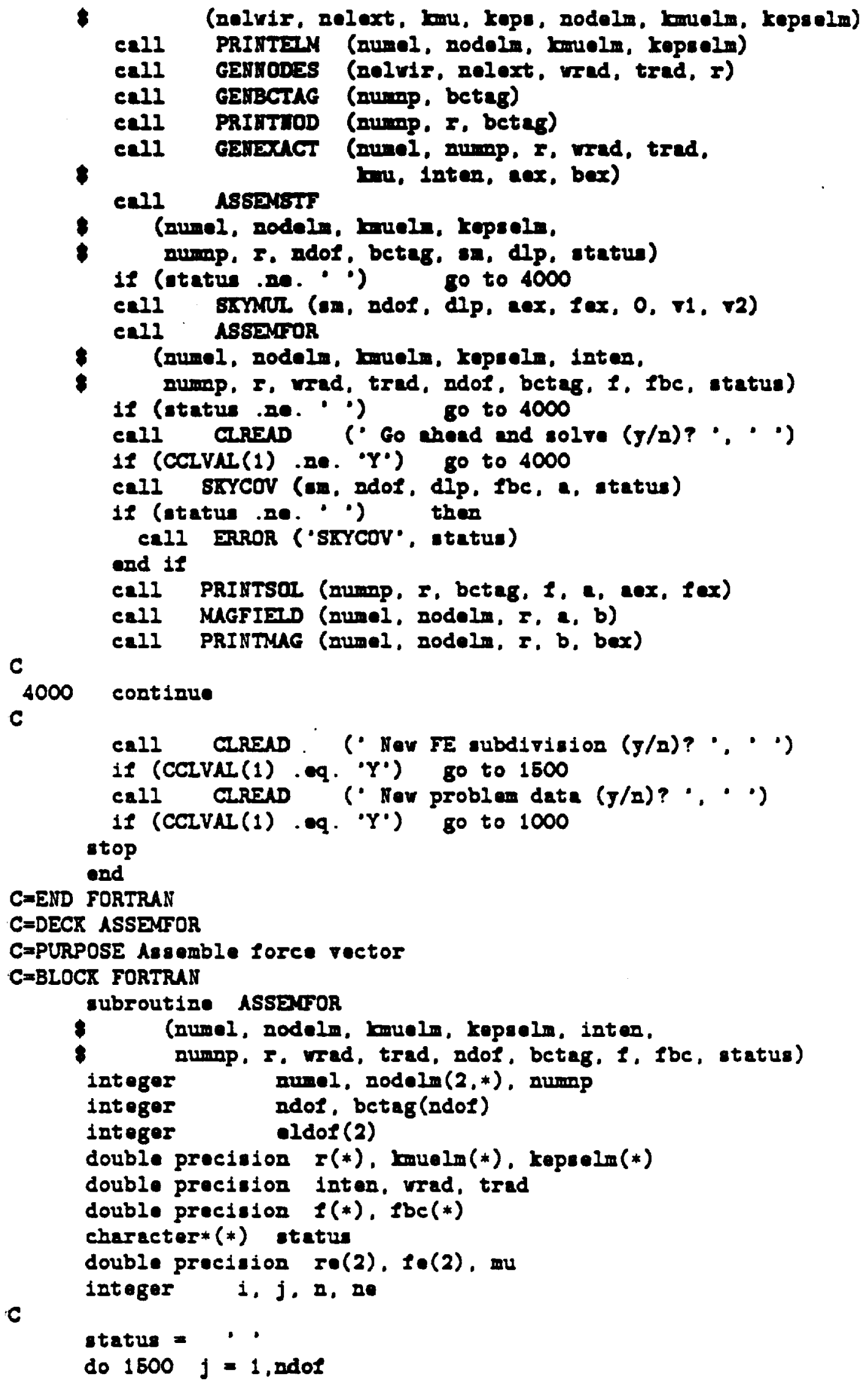




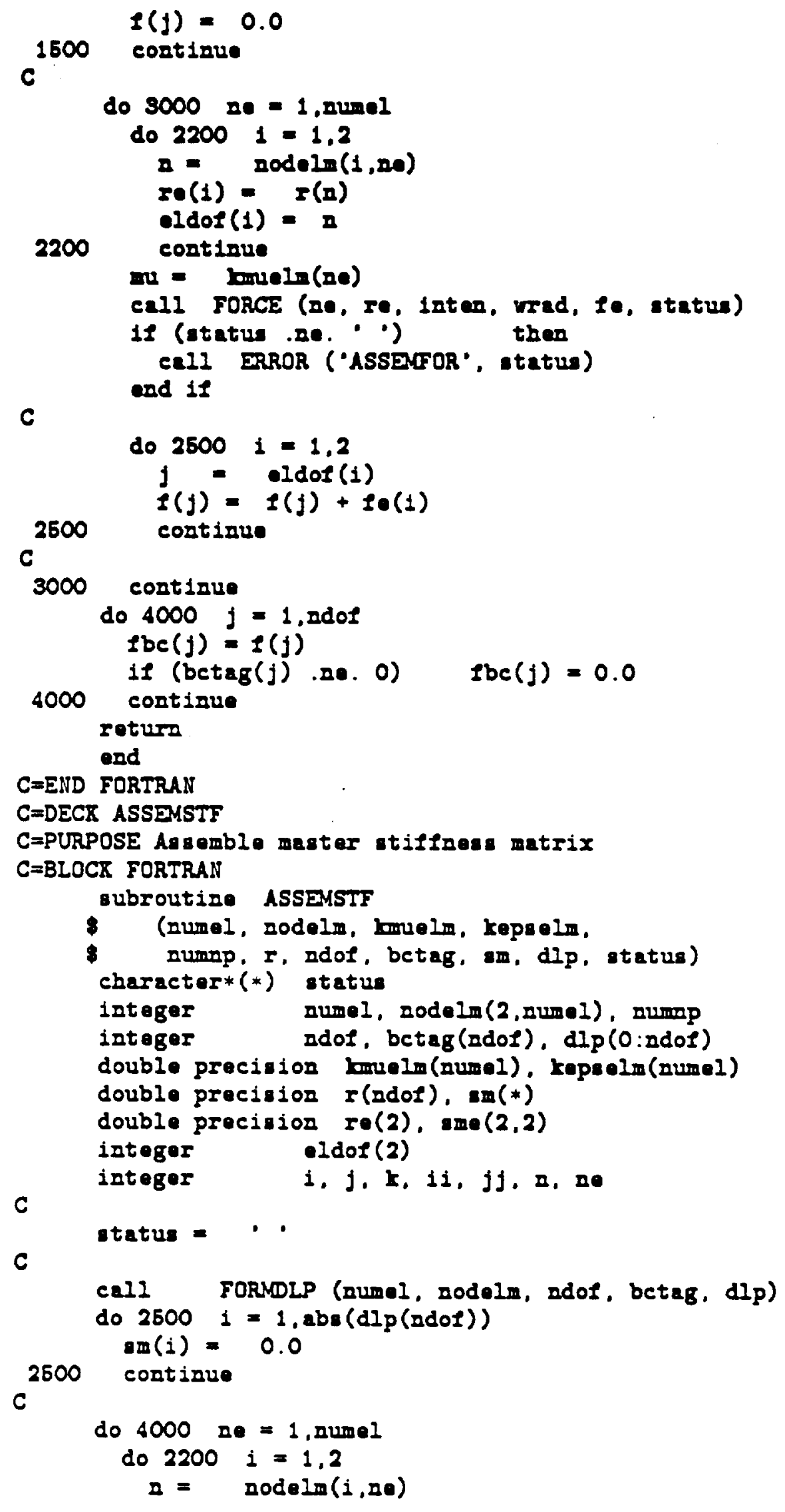




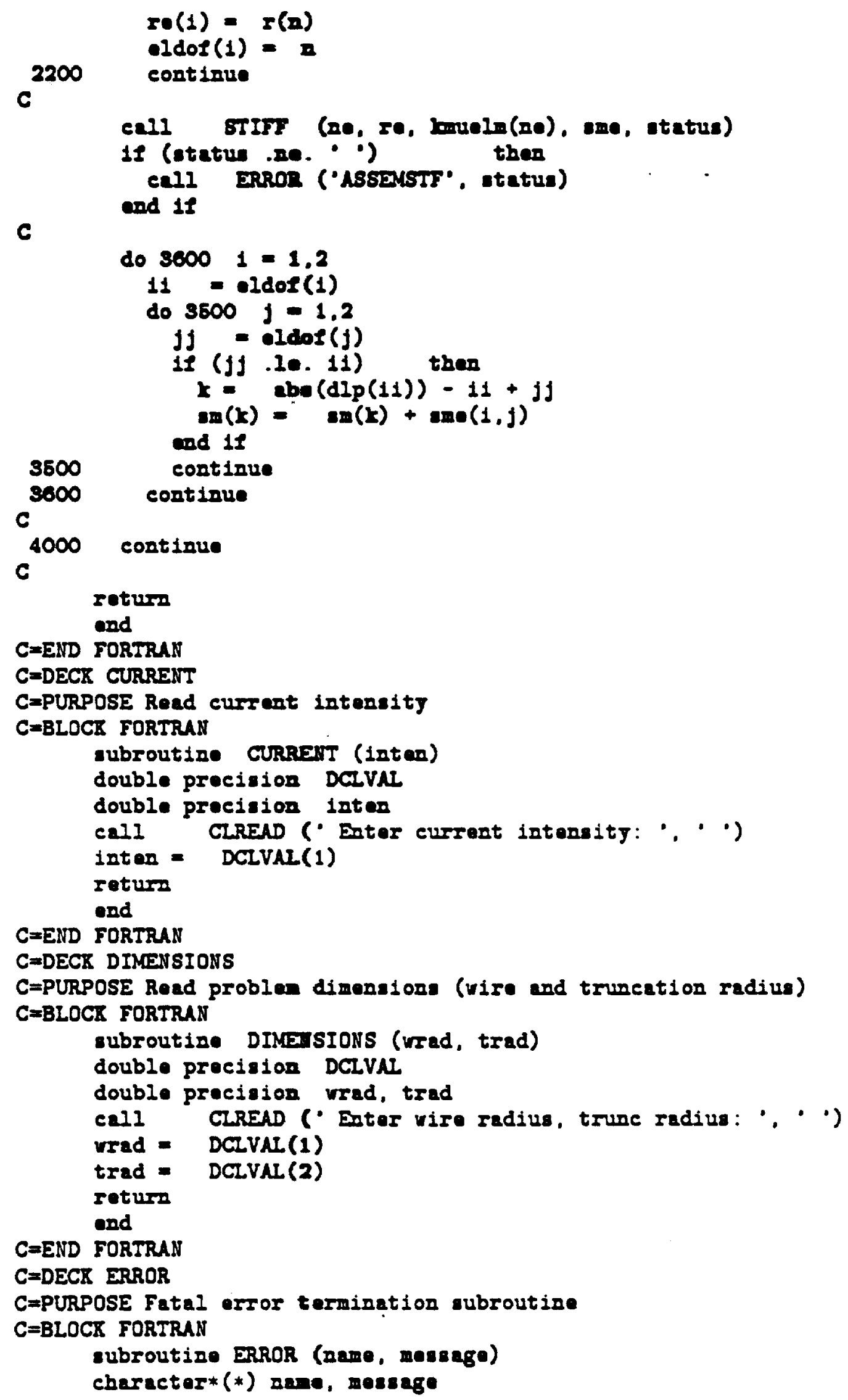




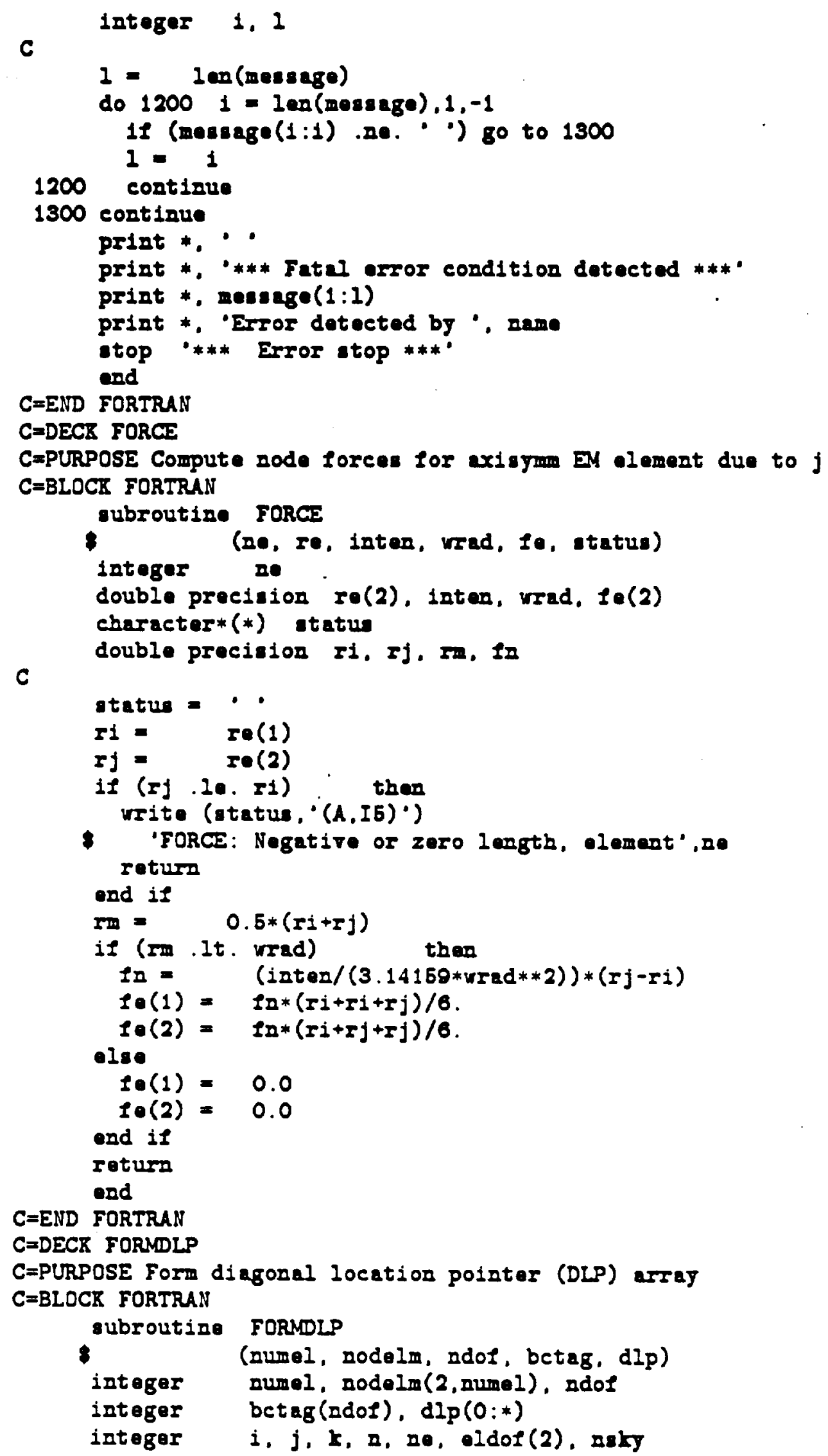


c

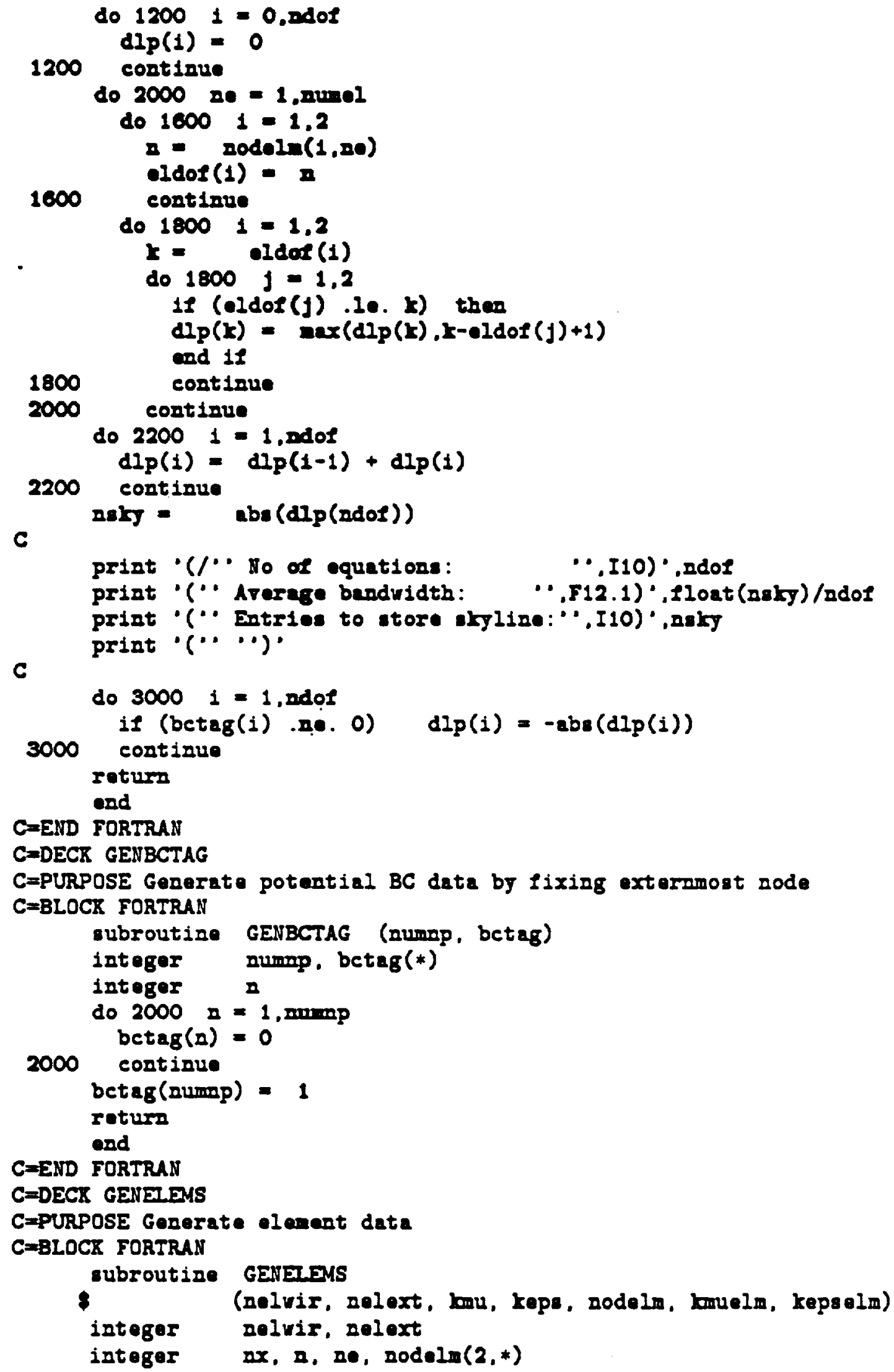




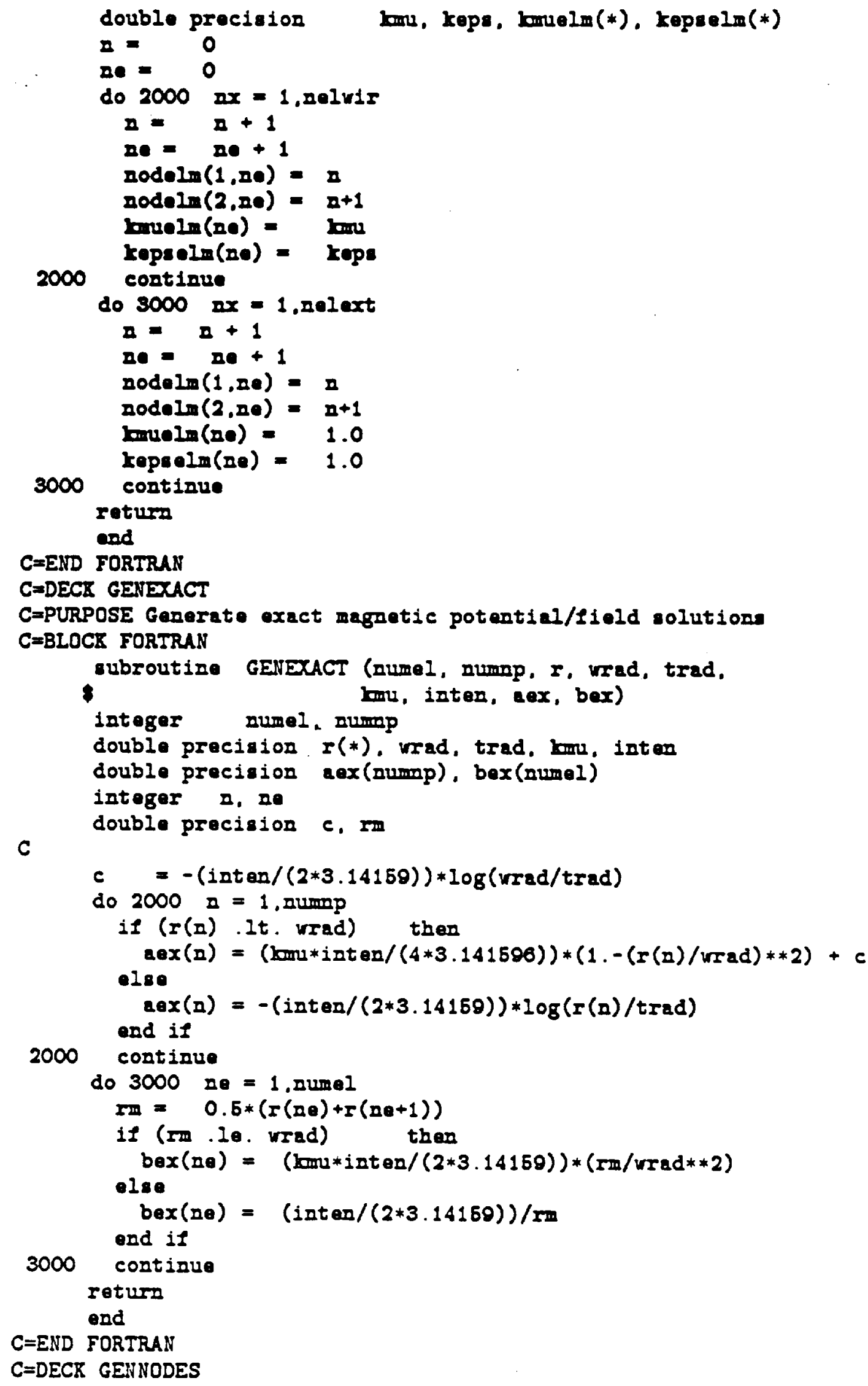




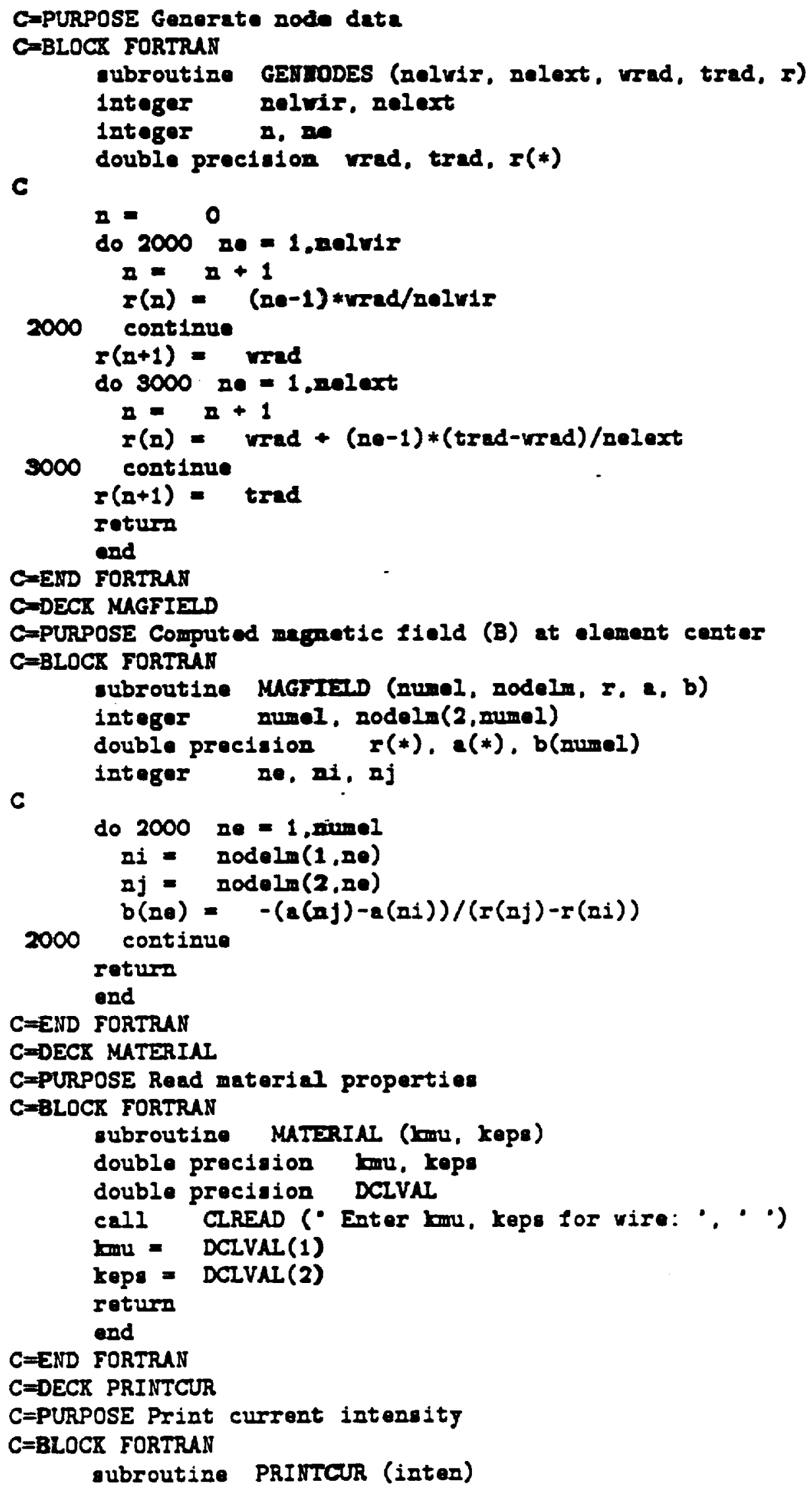




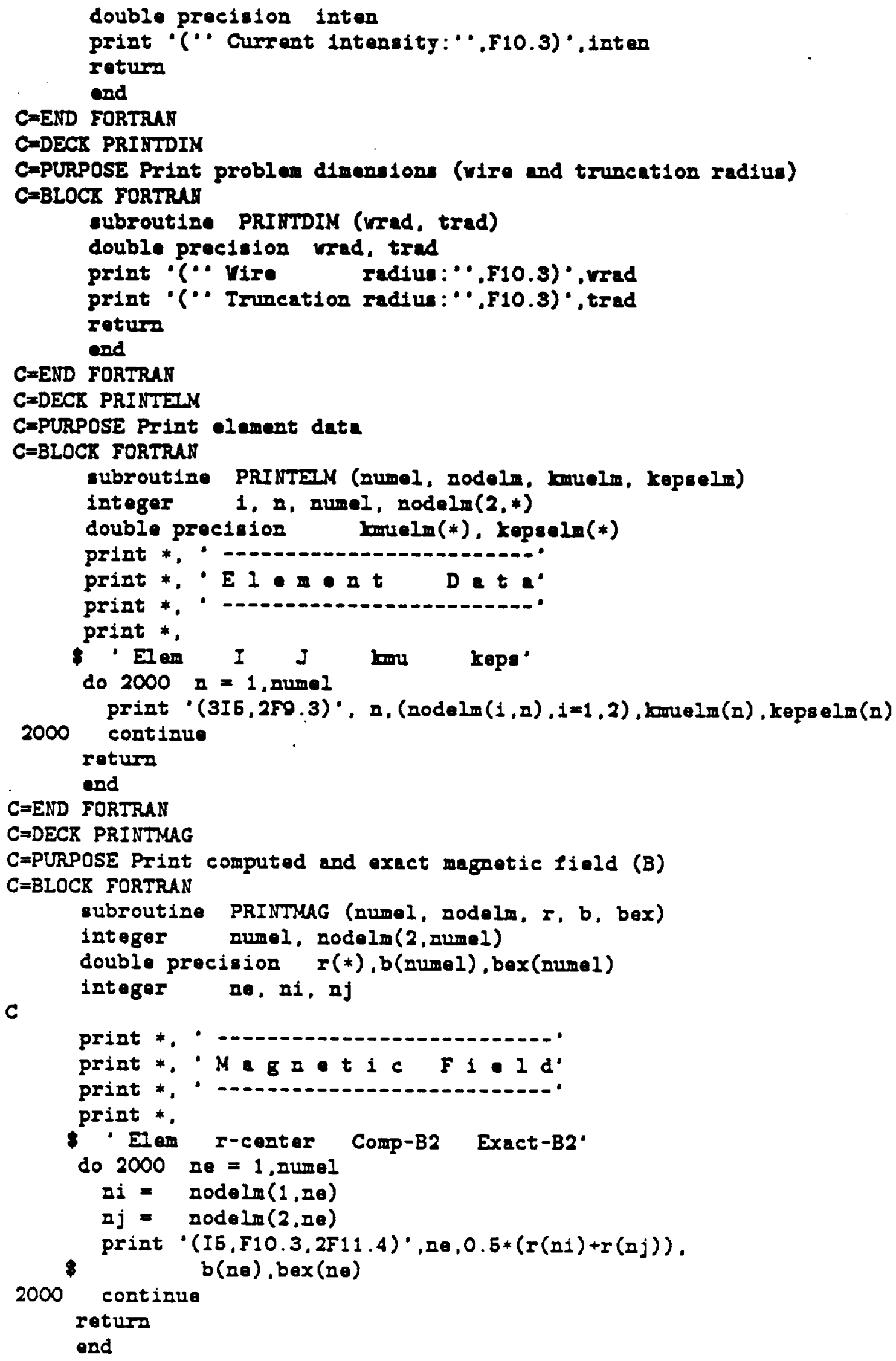




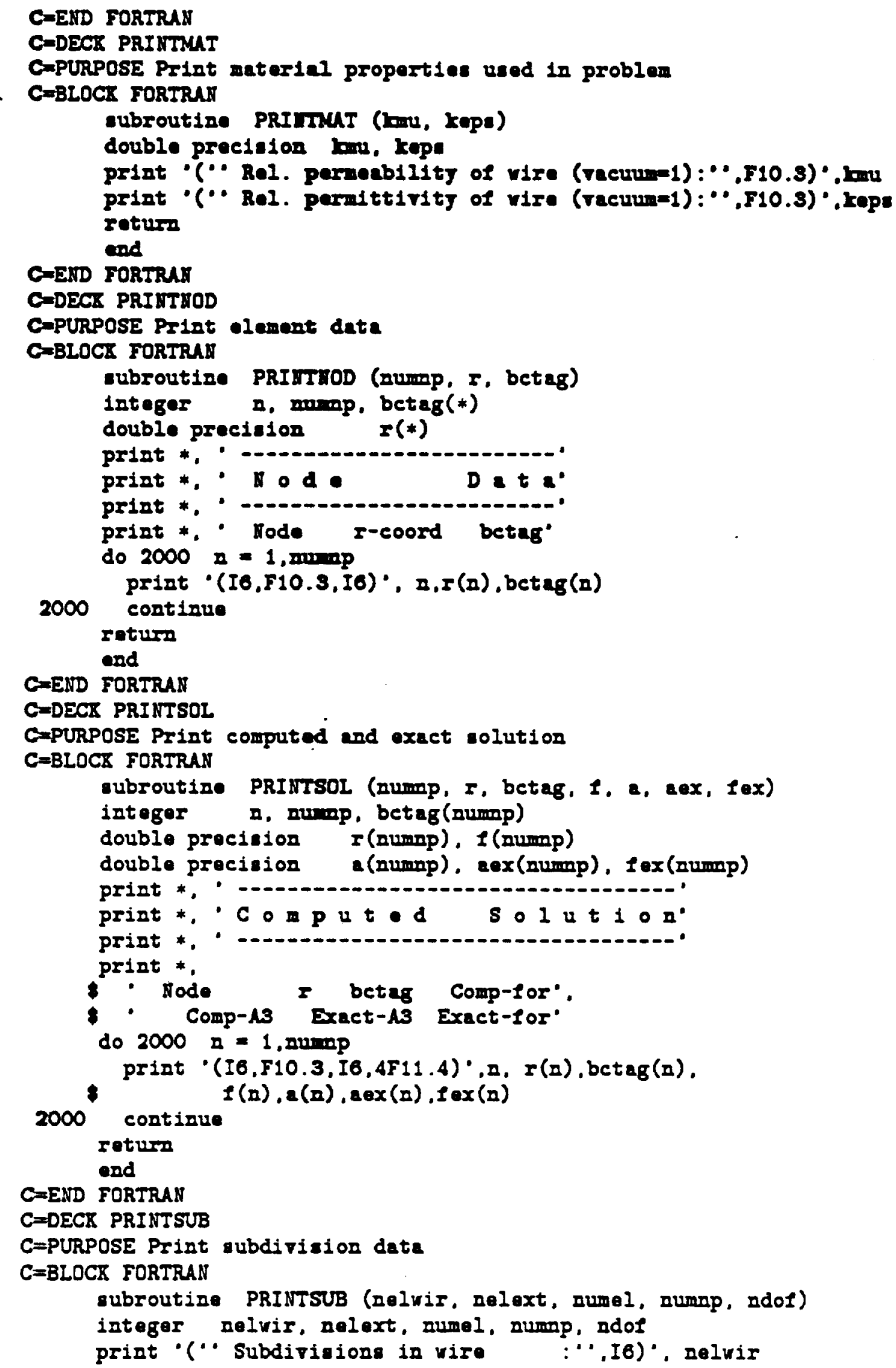




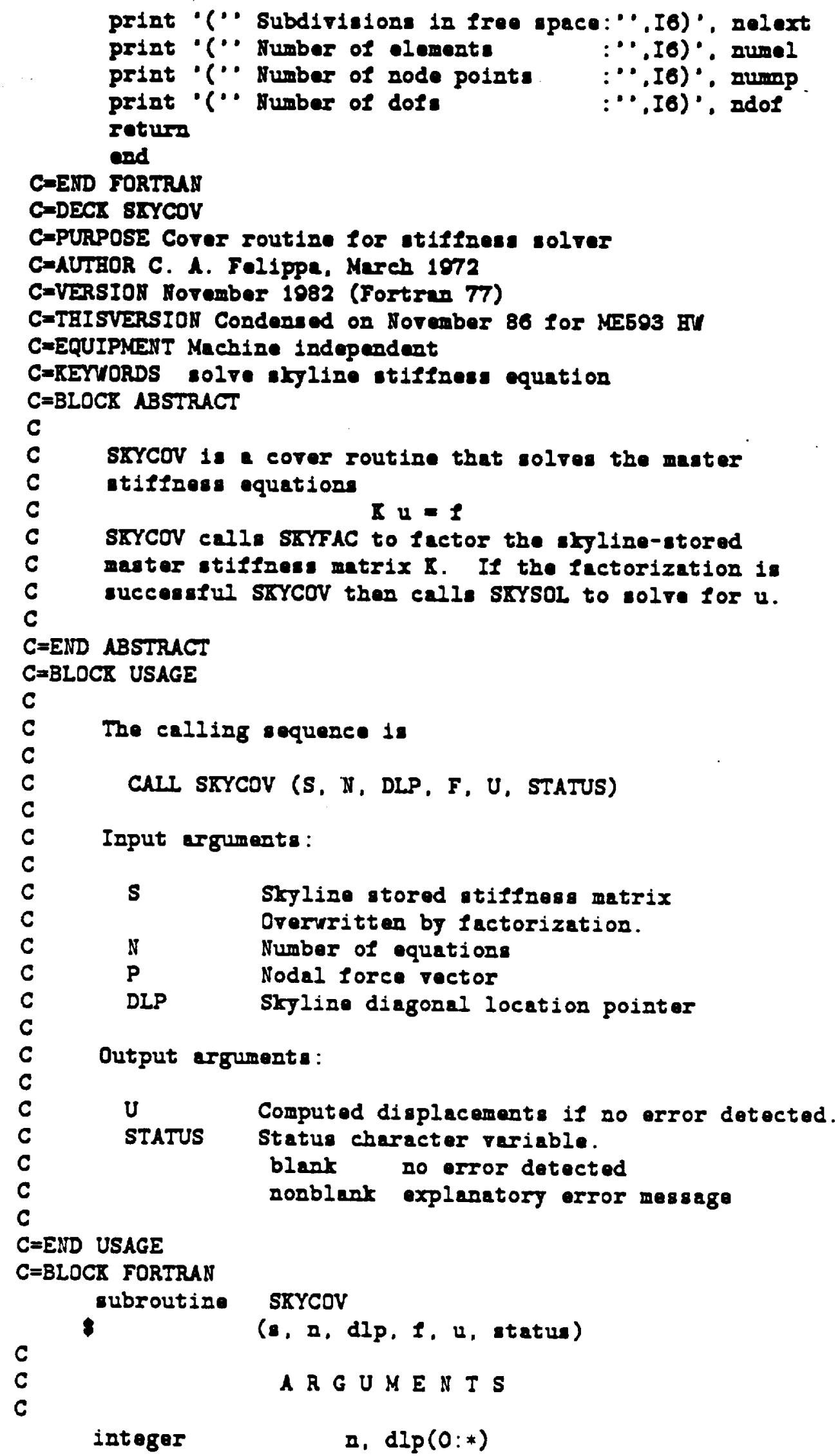




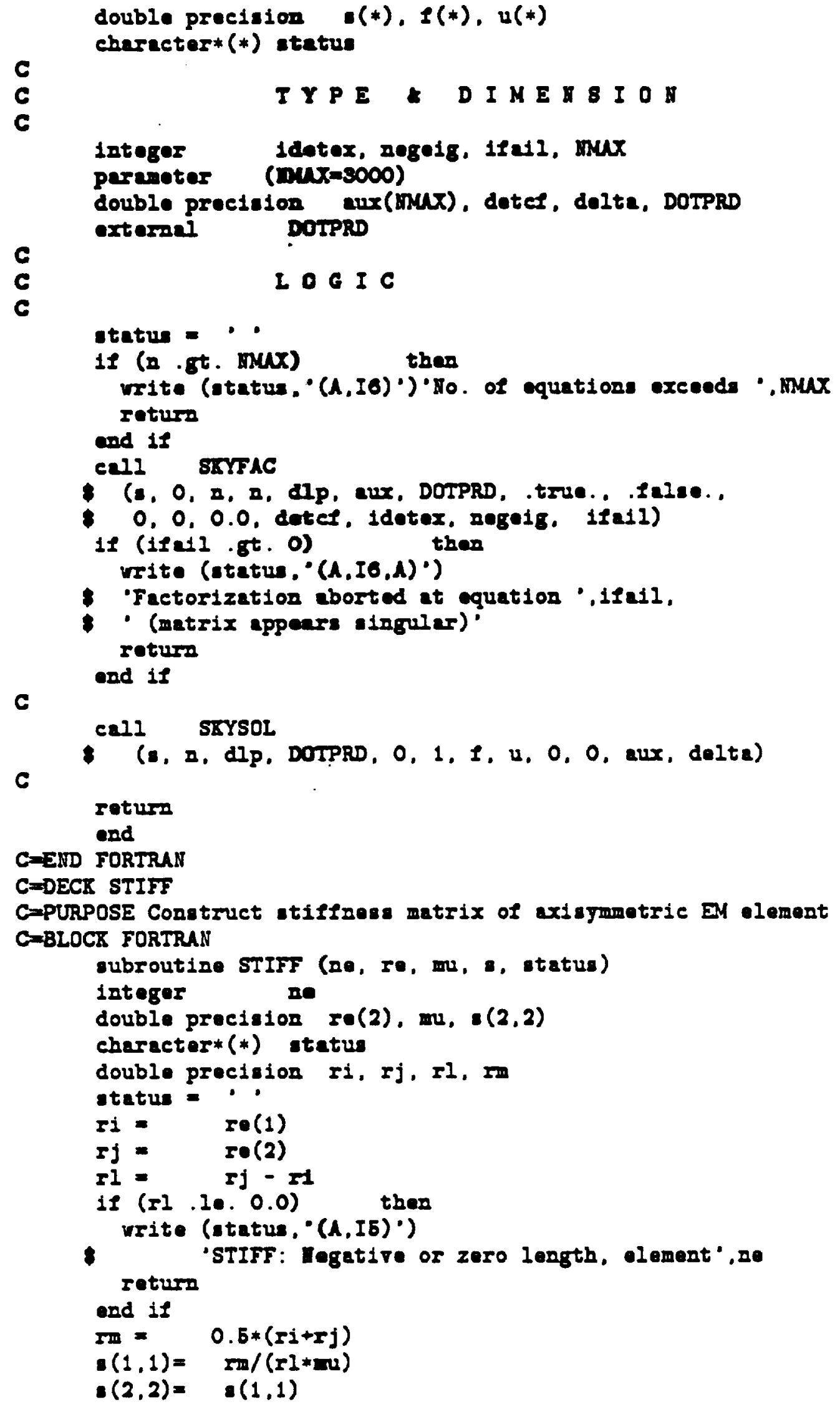




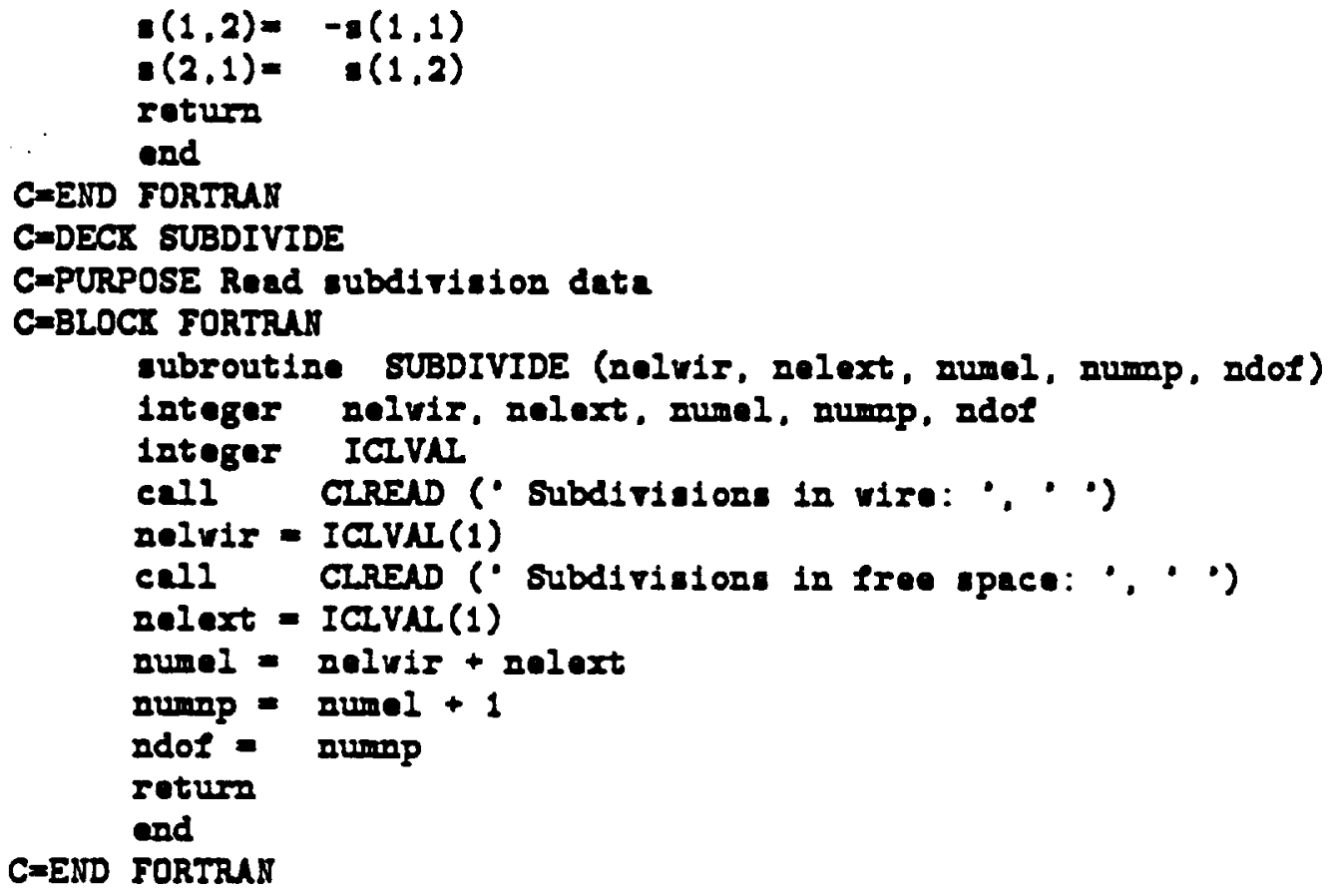



Public weporting burden for this collection of informeten is estimaled 10 average 1 hour per response, including the time for reviewing instructions, searching existing data sources, atheciong and maintaining the dala needed, and completing and reviewing the coliection of information. Send commenls regarding this burden estimate or any other aspect of this collection of inlormation, including suggestions tor Eucing this burden, to Washington Headquarters Sorvices, Directorate for information Operations and Reports, 1215 Jetferson Devie rtiotmay, Suite 1204, Artinglon, VA 22202-502, and to the Orlice of Management and Budget, Paperwork Peduction Project (0704-0188), Washington, DC 20503.

1. ACENCY USE ONLY (Leave blanK)

2. REPOAT DATE

November 1991
3. REPORT TYPE AND DATES COVERED

Final Contractor Report - Sept. 89

\section{TIRE AND SUBTITLE}

\section{FUNDING NUMBERS}

Electromagnetic Finite Elements Based on a Four-Potential

Variational Principle

\section{AUTHOR(S)}

James Schuler and Carlos A. Felippa

\section{PERFORMING ORGANIZATION NAME(S) AND ADDRESS(ES)}

University of Colorado

Department of Aerospace Engineering Sciences and

Center for Space Structures and Controls

WU- $505-63-5 B$

G-NAG3-934

Boulder, Colorado 80309

๑. STONSORING/MONITORING AGENCY MAMES(S) AND ADDRESS(ES)

National Aeronautics and Space Administration

Lewis Research Center

Cleveland, Ohio 44135-3191

9. performing organization REPORT NUMBER

None

10. SPONSORING/MONITORINC AGENCY REPORT NUMBEA

NASA CR -189067

11. DUPLEMENTARY NOTES

Project Manager, C.C. Chamis, Structures Division, NASA Lewis Research Center, (216) 433 - 3252.

12. DISTRIBUTION/AVAILABILITY STATEMENT

12b. DISTRIBUTION CODE

Unclassified - Unlimited

Subject Category 39

\section{AUSTAACT (Maximum 200 words)}

We derive electromagnetic finite elements based on a variational principle that uses the electromagnetic four-potential as primary variable. This choice is used to construct elements suitable for downstream coupling with mechanical and thermal finite elements for the analysis of electromagnetic/mechanical systems that involve superconductors. The key advantages of the four-potential are: the number of degrees of freedom per node remain modest as the problem dimensionality increases, jump discontinuities on interfaces are naturally accomodated, and static as well as dynamics are included without any a priori approximations. The new elements are tested on an axisymmetric problem under steady-state forcing conditions. The results are in excellent agreement with analytical solutions.

\begin{tabular}{|c|c|c|c|}
\hline \multirow{2}{*}{\multicolumn{3}{|c|}{$\begin{array}{l}\text { 14. SUBJECT TERMS } \\
\text { Mixed-fieled element; Four-potential; Down-stream coupling; Superconductors; Jump } \\
\text { discontinuties; Static; Dynamic; Error estimates; Application examples }\end{array}$}} & \multirow{2}{*}{\begin{tabular}{|c|} 
15. NUMBER OF PAGES \\
36 \\
16. PAICE CODE \\
A03 \\
\end{tabular}} \\
\hline & & & \\
\hline $\begin{array}{l}\text { 17. ECURITY CLASSIFICATION } \\
\text { OF REPORT } \\
\text { Unclassified }\end{array}$ & $\begin{array}{l}\text { 19. DECURITY CLASSIFICATION } \\
\text { OF THIS PAOE } \\
\text { Unclassified }\end{array}$ & $\begin{array}{l}\text { 19. SECUATY CLASSIFICATION } \\
\text { OF ABSTRACT } \\
\text { Unclassified }\end{array}$ & 20. LIMITATION OF ABSTRACT \\
\hline
\end{tabular}


